\title{
Local approximation from spline spaces on box meshes
}

\author{
Andrea Bressan . Tom Lyche
}

Received: date / Accepted: date

\begin{abstract}
This paper analyzes the approximation properties of spaces of piecewise tensor product polynomials over box meshes with a focus on application to IsoGeometric Analysis (IGA). Local and global error bounds with respect to Sobolev or reduced seminorms are provided. Attention is also paid to the dependence on the degree and exponential convergence is proved for the approximation of analytic functions in the absence of non-convex extended supports.
\end{abstract}

Keywords approximation · spline spaces · box meshes - quasi-interpolants · local error bounds · global error bounds · anisotropic error bounds · reduced seminorms $\cdot$ isogeometric analysis

Mathematics Subject Classification (2010) 41A15, 41A25, 41A63

\section{Introduction}

Let $\Omega$ be a union of axis aligned boxes in $\mathbb{R}^{n}$ and $\mathbb{S}$ a space of piecewise polynomials of degree $\boldsymbol{d}:=\left(d_{1}, \ldots, d_{n}\right)$ on $\Omega$. We use a local approximation operator $\aleph: L^{p}(\Omega) \rightarrow \mathbb{S}$, which reproduces polynomials of degree $\boldsymbol{d}$, to derive a priori approximation estimates for how well a function $f$ can be approximated by a function in $\mathbb{S}$. Bounds for the approximation error on both the mesh elements and on $\Omega$ are provided. The $L^{p}, L^{q}$ estimates on $\Omega$ have the following form

$$
\left\|\partial^{\boldsymbol{\sigma}}(f-\aleph f)\right\|_{p} \leq C \sum_{\boldsymbol{k} \in K}\left\|\rho_{\boldsymbol{k}} \partial^{\boldsymbol{k}} f\right\|_{q},
$$

Communicated by Nira Dyn

A. Bressan

Department of Mathematics, University of Pavia, via Ferrata 5, 27100 Pavia, Italia

E-mail: andrea.bressan@unipv.it

T. Lyche

Department of Mathematics, University of Oslo, P.O. box 1053, Blindern, 0316 Oslo, Norway Tel.: +47 22855888 E-mail: tom@math.uio.no 
where $K \subset \mathbb{N}^{n}$ is an index set of integers and the weights $\rho_{\boldsymbol{k}}$ are powers of the local resolution of $\mathbb{S}$. The precise form of the $\rho_{\boldsymbol{k}}$ depends on $K, \boldsymbol{k}, p, q, \boldsymbol{d}$ and $\boldsymbol{\sigma}$. The constant $C$ depends on a subset of some constants $C_{\lambda}, \ldots, C_{\mathbf{S}}$, which come from the abstract assumptions $\mathrm{H}_{\mathbb{P}} \ldots \mathrm{H}_{[\mathrm{S}}$, see Table 1 at the end of the paper.

Different index sets $K$ are possible, we focus on $K=\left\{\boldsymbol{k} \in \mathbb{N}^{n}:|\boldsymbol{k}|=d+1\right\}$ that correspond to Sobolev seminorms and on index sets $K$ that correspond to reduced seminorms that involve a smaller set of partial derivatives. For $\boldsymbol{\sigma}=\mathbf{0}$ the reduced seminorm leads to bounds in term of the partial derivatives in the coordinate directions

$$
\|f-\aleph f\|_{p} \leq C \sum_{i=1}^{n}\left\|\rho_{i} \partial_{i}^{k_{i}} f\right\|_{q} .
$$

For Sobolev seminorms and $p \leq q$ we are able to weaken the usual mesh quasi uniformity and mesh shape regularity assumptions matching those for tensor product splines. This can be seen by comparing our assumptions $\mathrm{H}_{\mathcal{M}}^{\#}$ and $\mathrm{H}_{\mathbf{S}}$ with the corresponding assumptions in 12 39 40 .

For $1 \leq p \leq q$ and $\boldsymbol{\sigma}=\mathbf{0}$ we obtain anisotropic estimates that take into consideration the local resolution of $\mathbb{S}$ in the different coordinate directions. In the other cases the estimates are isotropic, i.e., the directional information is discarded.

We also consider a sequence of spline spaces $\left\{\mathbb{S}_{d}\right\}$ of increasing degree $\boldsymbol{d}=$ $(d, \ldots, d)$ and corresponding approximation operators $\left\{\aleph_{d}\right\}$. Under suitable assumptions, if $f: \Omega \rightarrow \mathbb{R}$ admits an analytic extension on $\Delta \subset \mathbb{C}^{n}$, the approximation error decreases exponentially as $d$ increases. There are $\tau, C_{\tau}, \tau_{\#}, C_{\tau_{\#}} \in \mathbb{R}$ that depend on $\Delta, \sigma$ and $n$, but independent of the degree $d$, such that

$$
\begin{aligned}
\left\|\partial^{\sigma}\left(f-\aleph_{d} f\right)\right\|_{\infty} & \leq C_{\tau} \tau^{d+1-|\sigma|}\|f\|_{\infty, \Delta} \\
& \leq C_{\tau_{\#}} \tau_{\#}^{\left(\operatorname{dim} \mathbb{S}_{d}\right)^{\frac{1}{n}}}\|f\|_{\infty, \Delta} .
\end{aligned}
$$

Exponential convergence is of interest for the numerical solutions of PDEs and it is known to hold for Finite Element discretizations and univariate splines even in the presence of singularities [15]35. The novelty here is that exponential convergence is proved using local approximation operators and without assumptions on the smoothness of the functions in $\mathbb{S}_{d}$.

Following the application of splines to numerical methods for PDEs, mostly in the framework of IsoGeometric Analysis (IGA) [22, there has been a renewed interest in extension of tensor product splines (TPS) that are suitable to adaptive methods. Many of the available constructions fit our abstract framework. We provide an approximation operator $\aleph$ satisfying $\left[\mathrm{H}_{\mathbb{P}} \ldots \mathrm{H}_{[\mathbf{S}}\right.$ for TPS, Analysis Suitable T splines (AST) 4] 3], truncated hierarchical splines (THB) [20] and Locally Refined splines (LR) [16].

TPS are included for the sake of comparison and similar results are available in the literature 27 5] 6. The results for AST splines and for THB splines require weaker assumptions on the mesh compared to 39] 40] 3]; moreover, both global estimates and estimates without mixed derivatives are included. To the best of our knowledge the operator in Section 8.4 is the first quasi-interpolant that is proposed for LR splines.

The notation and the setting of this paper are described in Section 2 Section 3 contains the main approximation results under the assumptions $\mathrm{H}_{\mathbb{P}} \ldots \mathrm{H}_{\mathrm{s}}$, The 
index set $K$ of partial derivatives is determined by $\mathrm{H}_{\Pi}$ that describes polynomial approximation. In Section 4 we prove that different variants of averaged Taylor expansion operators satisfy $\mathrm{H}_{\Pi}$. Section 5 specializes the abstract theory to bounds in terms of Sobolev and reduced seminorms. Section 6 studies the approximation of analytic functions and contains the proof of exponential convergence. Section 7 recalls the B-spline theory necessary for this paper and provides the building blocks for the approximation operators $\aleph$ described in Section 8 . Sections 8 contains an approximation operator for TPS, AST, THB and LR. For each case we provide a definition of $\aleph$ that satisfies the required abstract assumptions. Section 9 contains a few closing remarks.

\section{Preliminaries}

Sets are usually denoted by capital letters, except when they are subsets of a topological space. The cardinality of a set $A$ is denoted by $\# A$. The interior of a subsets $\omega$ of a topological space, i.e. the biggest open set contained in $\omega$, is denoted by $\stackrel{\circ}{\omega}$ and the closure, i.e. the smallest closed set containing $\omega$, is denoted by $\bar{\omega}$.

$\mathbb{Z}$ is the set of integers and $\mathbb{N}:=\{0,1, \ldots\}$. The floor, or integer part, of a real number $a \in \mathbb{R}$ is denoted by $\lfloor a\rfloor:=\max \{z \in \mathbb{Z}: z \leq a\}$. We also use the positive and negative parts: $a_{+}:=\max \{0, a\}$ and $a_{-}:=\min \{0, a\}$ so that $a=a_{+}+a_{-}$.

\subsection{Multi-index notation}

Multi-indices in $\mathbb{N}^{n}$ and vectors in $\mathbb{R}^{n}$ are highlighted by boldface. For convenience we use $\mathbf{0}:=(0, \ldots, 0)$ and $\mathbf{1}:=(1, \ldots, 1)$ in $\mathbb{N}^{n}$ and $\mathbb{R}^{n}$. Given a multi-index $\boldsymbol{\alpha} \in \mathbb{N}^{n}$ and a sufficiently smooth function $f: \Omega \subseteq \mathbb{R}^{n} \rightarrow \mathbb{R}$

$$
\partial^{\alpha} f:=\frac{\partial^{\alpha_{1}+\cdots+\alpha_{n}}}{\partial x_{1}^{\alpha_{1}} \cdots \partial x_{n}^{\alpha_{n}}} f .
$$

The factorial of a multi-index $\boldsymbol{\alpha} \in \mathbb{N}^{n}$ is $\boldsymbol{\alpha} !:=\prod_{i=1}^{n}\left(\alpha_{i} !\right)$. Consequently for $\boldsymbol{\alpha}, \boldsymbol{\beta} \in$ $\mathbb{N}^{n}$ we have

$$
\left(\begin{array}{c}
\boldsymbol{\alpha} \\
\boldsymbol{\beta}
\end{array}\right):=\frac{\boldsymbol{\alpha} !}{(\boldsymbol{\alpha}-\boldsymbol{\beta}) ! \boldsymbol{\beta} !}=\prod_{i=1}^{n}\left(\begin{array}{c}
\alpha_{i} \\
\beta_{i}
\end{array}\right) .
$$

Many scalar operations are extended to vectors in $\mathbb{R}^{n}$. The relations $<,>, \geq, \leq$ hold on a pair of vectors $\boldsymbol{a}=\left(a_{1}, \ldots, a_{n}\right), \boldsymbol{b}=\left(b_{1}, \ldots, b_{n}\right) \in \mathbb{R}^{n}$ if and only if they hold for each pair of components, e.g.

$$
\boldsymbol{a} \leq \boldsymbol{b} \Leftrightarrow a_{i} \leq b_{i}, \forall i=1, \ldots, n .
$$

Similarly $\max \{\boldsymbol{a}, \boldsymbol{b}\}, \min \{\boldsymbol{a}, \boldsymbol{b}\}$, and $\boldsymbol{a}_{ \pm}$act component wise: e.g.,

$$
\boldsymbol{a}_{+}:=\left(\left(a_{1}\right)_{+}, \ldots,\left(a_{n}\right)_{+}\right), \quad \max \{\boldsymbol{a}, \boldsymbol{b}\}:=\left(\max \left\{a_{1}, b_{1}\right\}, \ldots, \max \left\{a_{n}, b_{n}\right\}\right) .
$$

For a vector $\boldsymbol{a} \in \mathbb{R}^{n}$ we define

$$
\begin{array}{lll}
|\boldsymbol{a}|:=\sum_{i=1}^{n}\left|a_{i}\right|, & \|\boldsymbol{a}\|:=\left(\sum_{i=1}^{n} a_{i}^{2}\right)^{\frac{1}{2}}, \\
\max \boldsymbol{a}:=\max \left\{a_{1}, \ldots, a_{n}\right\}, & \min \boldsymbol{a}:=\min \left\{a_{1}, \ldots, a_{n}\right\},
\end{array}
$$


and for $\boldsymbol{a}, \boldsymbol{b} \in \mathbb{R}^{n}$, we define the power $\boldsymbol{a}^{\boldsymbol{b}}$ as

$$
\boldsymbol{a}^{\boldsymbol{b}}:=\prod_{i=1}^{n} a_{i}^{b_{i}}
$$

Note that we have the following relations for $\boldsymbol{a}, \boldsymbol{b}, \boldsymbol{c} \in \mathbb{R}^{n}$

$$
\frac{\boldsymbol{a}^{\boldsymbol{c}}}{\boldsymbol{b}^{\boldsymbol{c}}}=\left(\frac{a_{1}}{b_{1}}, \ldots, \frac{a_{n}}{b_{n}}\right)^{\boldsymbol{c}}, \quad \boldsymbol{a} \geq \mathbf{0} \Rightarrow \boldsymbol{a}^{\boldsymbol{b}} \leq(\max \boldsymbol{a})^{\left|\boldsymbol{b}_{+}\right|}(\min \boldsymbol{a})^{-\left|\boldsymbol{b}_{-}\right|} .
$$

\subsection{Function spaces}

As usual $\mathcal{C}(U)$ denotes the space of continuous functions $U \rightarrow \mathbb{R}$ and for $\alpha \in \mathbb{N}$, $\mathcal{C}^{\alpha}(U)$ is the space of continuous functions whose derivatives up to order $\alpha$ are in $\mathcal{C}(U)$. The definition is extended to $\boldsymbol{\alpha} \in \mathbb{N}^{n}$ with the following meaning

$$
\mathcal{C}^{\boldsymbol{\alpha}}(U):=\left\{f: U \rightarrow \mathbb{R}: \forall \boldsymbol{\beta} \in \mathbb{N}^{n} \text { with } \boldsymbol{\beta} \leq \boldsymbol{\alpha}, \partial^{\boldsymbol{\beta}} f \in \mathcal{C}(U)\right\}
$$

Polynomials of maximal degree $d$ and polynomials of multidegree $\boldsymbol{d}=\left(d_{1}, \ldots, d_{n}\right)$ are respectively denoted by

$$
\mathbb{P}_{d}:=\operatorname{span}\left\{\boldsymbol{x}^{\boldsymbol{\alpha}}: \boldsymbol{\alpha} \in \mathbb{N}^{n},|\boldsymbol{\alpha}| \leq d\right\}, \quad \mathbb{P}_{\boldsymbol{d}}:=\operatorname{span}\left\{\boldsymbol{x}^{\boldsymbol{\alpha}}: \boldsymbol{\alpha} \in \mathbb{N}^{n}, \boldsymbol{\alpha} \leq \boldsymbol{d}\right\}
$$

In general the polynomial space $\mathbb{P}_{A}$ associated with $A \subseteq \mathbb{N}^{n}$ is

$$
\mathbb{P}_{A}:=\operatorname{span}\left\{\boldsymbol{x}^{\alpha}: \boldsymbol{\alpha} \in A\right\}
$$

The standard Lebesgue measure on $\mathbb{R}^{n}$ is denoted by $\mu$. As customary $L^{p}(U)$, $1 \leq p \leq \infty$, is the Banach space of the equivalence classes of measurable functions $U \rightarrow \mathbb{R}$ that agree almost everywhere (a.e.) and $L^{p}\left(U ; \mathbb{R}^{n}\right)$ is the corresponding space of vector valued functions. The norm on $L^{p}(U)$ is the usual

$$
\|f\|_{p, U}:= \begin{cases}\left(\int_{U}|f(\boldsymbol{x})|^{p} d \boldsymbol{x}\right)^{\frac{1}{p}} & p \neq \infty \\ \operatorname{ess} \sup \{|f(\boldsymbol{x})|, \boldsymbol{x} \in U\} & p=\infty\end{cases}
$$

The dual of $L^{p}(U)$ is the space of linear continuous functionals $\lambda: L^{p}(U) \rightarrow \mathbb{R}$ and it is denoted by $L^{p}(U)^{*}$. It is a Banach space with norm

$$
\|\lambda\|_{* p}:=\sup \left\{\lambda(f): f \in L^{p}(U):\|f\|_{p}=1\right\} .
$$

If $1<p<\infty$ then $L^{p}(U)^{*}$ is isometrically isomorphic to $L^{p^{\prime}}(U)$ where $p^{\prime}=$ $(1-1 / p)^{-1}$ and the isomorphism maps $\lambda$ to $w$ if for all $f \in L^{p}(U)$

$$
\lambda(f)=\int_{U} f(\boldsymbol{x}) w(\boldsymbol{x}) d \boldsymbol{x} .
$$

The support of a function $f \in L^{p}(U)$ is the closed set

$$
\operatorname{supp} f:=U \backslash \bigcup_{W \in Z} W, \quad Z=\left\{\text { open } W \subseteq U:\left.f\right|_{W}=0 \text { a.e. }\right\} \text {. }
$$


This definition agrees with the standard definition supp $f=\overline{\{\boldsymbol{x} \in U: \tilde{f}(\boldsymbol{x}) \neq 0\}}$ if $\tilde{f}$ is a continuous representative of $f$. For $\lambda \in L^{p}(U)^{*}$ we use $\operatorname{supp} \lambda$ to denote the distributional support, i.e.

$$
\operatorname{supp} \lambda:=U \backslash \bigcup_{W \in Z} W, Z=\{\text { open } W \subseteq U: \forall f \text { with } \operatorname{supp} f \subset W, \lambda(f)=0\} .
$$

For $1<p<\infty$ the supports of $\lambda$ as an element of $L^{p}(U)^{*}$ and as an element of $L^{p^{\prime}}(U)$ coincide.

To deal with mixed norms as in (1) we introduce the following notations

$$
\nu:=\frac{1}{p}-\frac{1}{q}, \quad \nu:=(\nu, \ldots, \nu)
$$

\subsection{Spline spaces on box meshes}

In this paper a box in $\mathbb{R}^{n}$ is axis aligned, i.e. it is either empty or a Cartesian product of non-empty closed intervals $\eta=[\boldsymbol{a}, \boldsymbol{b}]:=\left[a_{1}, b_{1}\right] \times \cdots \times\left[a_{n}, b_{n}\right]$. Given a set $U \subset \mathbb{R}^{n}$ the bounding box of $U$ is denoted $\mathrm{b}(U)$ and it is the smallest box containing $U$ :

$$
\mathrm{b}(U):=\min \{\text { box } \eta: U \subseteq \eta\} .
$$

The size of a box $\eta=[\boldsymbol{a}, \boldsymbol{b}]=\left[a_{1}, b_{1}\right] \times \cdots \times\left[a_{n}, b_{n}\right]$ is the vector

$$
\boldsymbol{h}_{\eta}:=\left(h_{\eta, 1}, \ldots, h_{\eta, n}\right):=\left(b_{1}-a_{1}, \ldots, b_{n}-a_{n}\right) \in \mathbb{R}^{n} .
$$

For a set $U \subset \mathbb{R}^{n}$ we define $\boldsymbol{h}_{U}:=\boldsymbol{h}_{\mathrm{b}(U)}$. For all $U \subset \mathbb{R}^{n}$ we have

$$
\mu(U) \leq \boldsymbol{h}_{U}^{\mathbf{1}}=\prod_{i=1}^{n} h_{U, i}=\mu(\mathrm{b}(U)) .
$$

A box mesh $\mathcal{M}$ is a collection of boxes having non-empty pairwise-disjoint interiors

$$
\omega \in \mathcal{M} \Rightarrow \stackrel{\circ}{\omega} \neq \emptyset, \quad \omega, \eta \in \mathcal{M}, \omega \neq \eta \Rightarrow \stackrel{\circ}{\cap} \stackrel{\circ}{\eta}=\emptyset .
$$

The domain $\Omega$ of the box mesh $\mathcal{M}$ is the union of the boxes it contains

$$
\Omega:=\bigcup_{\omega \in \mathcal{M}} \omega
$$

Note that the domain of a box mesh is not necessarily connected. We will shorten $\|\cdot\|_{p, \Omega}$ to $\|\cdot\|_{p}$, i.e. the domain $\Omega$ will be subsumed in norms.

A spline space $\mathbb{S}$ of degree $\boldsymbol{d}$ on a box mesh $\mathcal{M}$ is a subspace of

$$
\left\{f: \Omega \rightarrow \mathbb{R}: \forall \omega \in \mathcal{M}, \exists g \in \mathbb{P}_{\boldsymbol{d}}: f=g \text { in } \stackrel{\circ}{\omega}\right\} .
$$

We always assume $\mathbb{P}_{\boldsymbol{d}} \subseteq \mathbb{S}$. The support of any function $f \in \mathbb{S}$ is a union of boxes in $\mathcal{M}$. We denote the set of boxes contained in supp $f$ by

$$
\mathrm{M}_{f}:=\{\omega \in \mathcal{M}: \omega \subseteq \operatorname{supp} f\}
$$




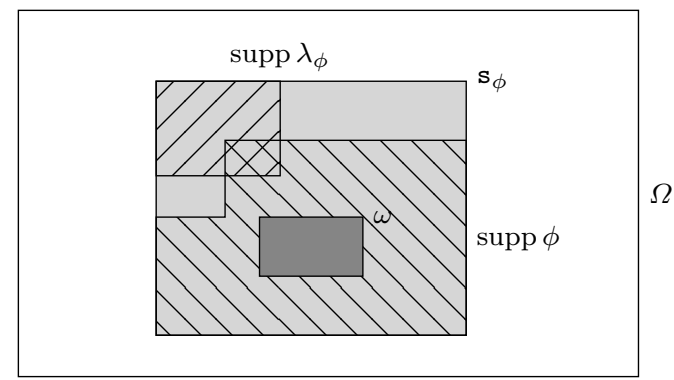

Fig. 1 An example of $\omega(\square), \operatorname{supp} \phi(\), \operatorname{supp} \lambda_{\phi}(/ /)$ and $\mathbf{s}_{\phi}(\quad)$.

\subsection{Quasi-interpolants}

A quasi-interpolant $\aleph: L^{p}(\Omega) \rightarrow \mathbb{S}$ can be constructed using a generating system $\Phi$ and a collection of linear functionals $\Lambda=\left\{\lambda_{\phi}, \phi \in \Phi\right\} \subset L^{p}(\Omega)^{*}$ by setting

$$
\aleph f:=\sum_{\phi \in \Phi} \lambda_{\phi}(f) \phi .
$$

Note that $\aleph$ is linear, i.e., $\aleph(a f+b g)=a \aleph f+b \aleph f$.

For $U \subseteq \Omega$ we define the set $\mathrm{A}_{U}$ of active generators of $U$ as

$$
\mathrm{A}_{U}:=\{\phi \in \Phi: \operatorname{supp} \phi \cap \stackrel{\circ}{U} \neq \emptyset\}
$$

In particular we will use $\mathrm{A}_{\omega}$ for $\omega \in \mathcal{M}$. To allow for $\operatorname{supp} \lambda_{\phi} \not \subseteq \operatorname{supp} \phi$ we introduce the extended support $\mathbf{s}_{\phi}$ and the set $\mathrm{E}_{\omega}$ of extended active generators of $\omega$

$$
\mathbf{s}_{\phi}:=\mathrm{b}\left(\operatorname{supp} \phi \cup \operatorname{supp} \lambda_{\phi}\right) \cap \Omega, \quad \mathrm{E}_{\omega}:=\left\{\phi \in \Phi: \omega \subseteq \mathbf{s}_{\phi}\right\} .
$$

Note that $\mathrm{E}_{\omega} \supseteq \mathrm{A}_{\omega}$ and the equality holds if $\operatorname{supp} \lambda_{\phi} \subseteq \operatorname{supp} \phi$. An example of $\mathbf{s}_{\phi}$ is shown in Fig. 1. The set $\mathbf{s}_{\phi}$ is convex and a box if and only if $\mathrm{b}\left(\operatorname{supp} \phi \cup \operatorname{supp} \lambda_{\phi}\right) \subseteq$ $\Omega$. In this paper we assume that the $\mathbf{s}_{\phi}$ is a truncated box, i.e. it has the form

$$
\mathbf{s}_{\phi}=\overline{\eta \backslash \beta}
$$

where $\eta, \beta$ are two boxes in $\mathbb{R}^{n}$ sharing a common vertex and $\beta \subset \eta$. The truncated boxes in $\mathbb{R}^{n}$ are classified by the face of $\eta$ that does not intersect $\mathbf{s}_{\phi}$ and has maximum dimension $m, m:=-1$ when $\mathbf{s}_{\phi}=\eta$. It can be shown that there are $3^{n}-2 n$ types of truncated boxes in $\mathbb{R}^{n}$. In $\mathbb{R}^{2}$ a truncated box is either a rectangle or an L-shaped domain, see Fig. 2 In $\mathbb{R}^{3}$ it is either a box, a Fichera corner or a product of an L-shape and an interval, see Fig. 3.

Diversification, see [33], can sometimes provide a $\Phi$ such that all the $\mathbf{s}_{\phi}$ 's are truncated boxes, starting from a $\Psi$ not having this property. It consists in replacing each generator $\phi \in \Psi$ with the set of its restrictions to the connected components of $\operatorname{supp} \phi$. A similar technique is decoupling, see [28] and the variation in [11]. In Fig. 4 there are three examples of a non-convex $\mathbf{s}_{\phi}$ in $\mathbb{R}^{2}$. The left is a truncated box. The middle one is not a truncated box, but diversification of $\phi$ can provide two truncated boxes. The right one is not a truncated box and neither diversification, nor decoupling can be applied. 


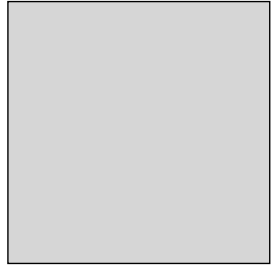

1 type, $(m=-1)$

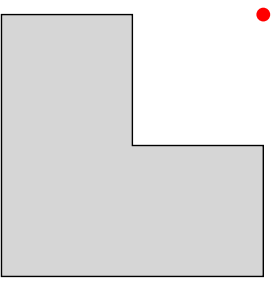

4 types, $(m=0)$

Fig. 2 Truncated boxes in $\mathbb{R}^{2}$ : the box and the L-shaped domains. The face of $\eta$ of dimension $m$ not intersecting $\mathbf{s}_{\phi}$ is highlighted in red.

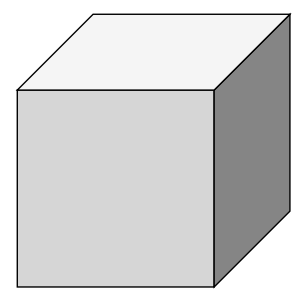

1 type, $(m=-1)$

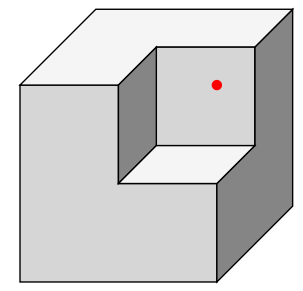

8 types, $(m=0)$

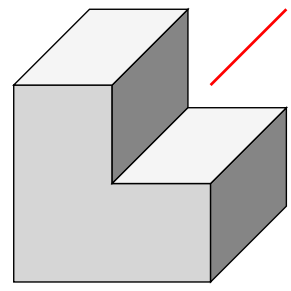

12 types, $(m=1)$

Fig. 3 Truncated boxes in $\mathbb{R}^{3}$ : the box, the Fichera corner and the product of an L-shape with an interval. The face of $\eta$ of dimension $m$ not intersecting $\mathbf{s}_{\phi}$ is highlighted in red.
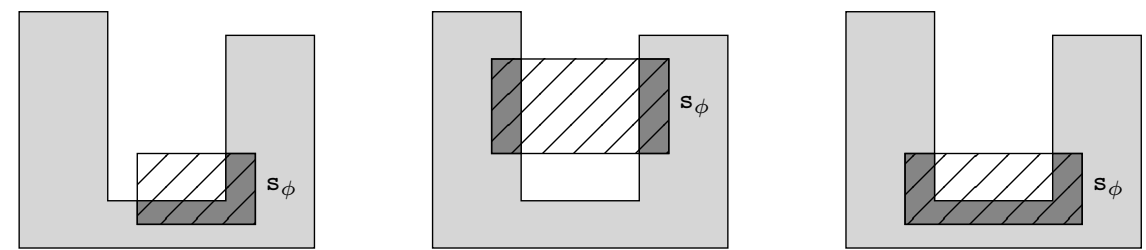

Fig. 4 Three examples of a non-convex $\mathbf{s}_{\phi}(\square)$, where $b\left(\mathbf{s}_{\phi}\right)(/ /)$ is not contained in the U shaped region $\Omega(\quad)$.

We describe the approximation power of a spline space using the piecewise constant functions $\mathrm{h}_{\Phi}$ and $\mathrm{h}_{\mathcal{M}}$ in $L^{\infty}\left(\Omega, \mathbb{R}^{n}\right)$ defined as follows:

$$
\begin{aligned}
& \left.\mathrm{h}_{\Phi}\right|_{\omega}:=\left.\left(\mathrm{h}_{\Phi, 1}, \ldots, \mathrm{h}_{\Phi, n}\right)\right|_{\omega}:=\max _{\phi \in \mathbb{E}_{\omega}}\left\{\boldsymbol{h}_{\mathrm{s}_{\phi}}\right\} \\
& \left.\mathrm{h}_{\mathcal{M}}\right|_{\omega}:=\left.\left(\mathrm{h}_{\mathcal{M}, 1}, \ldots, \mathrm{h}_{\mathcal{M}, n}\right)\right|_{\omega}:=\boldsymbol{h}_{\omega}
\end{aligned}
$$

The function $\mathrm{h}_{\Phi}$ gives a local measure of the resolution of $\mathbb{S}$, while $\mathrm{h}_{\mathcal{M}}$ represents the local mesh size. The form of $\rho_{\boldsymbol{k}}$ in (1) classifies the estimates as

- anisotropic, if $\rho_{\boldsymbol{k}}=\mathrm{h}_{\Phi}^{e_{\boldsymbol{k}}}$ with $e_{\boldsymbol{k}} \in \mathbb{R}^{n}$

- isotropic, if $\rho_{\boldsymbol{k}}=\left(\max \mathrm{h}_{\Phi}\right)^{e_{\boldsymbol{k}}}$ with $e_{\boldsymbol{k}} \in \mathbb{R}$.

Anisotropic estimates differentiate between the resolution in each coordinate directions, while for isotropic estimates only the maximum is considered. 
2.5 Some useful inequalities

For all $a_{1}, \ldots, a_{m} \geq 0$ and $e \geq 0$ we have

$$
\begin{aligned}
\left(\sum_{i=1}^{m} a_{i}\right)^{e} & \leq \sum_{i=1}^{m} a_{i}^{e} \leq m^{1-e}\left(\sum_{i=1}^{m} a_{i}\right)^{e}, \quad 0 \leq e \leq 1, \\
\sum_{i=1}^{m} a_{i}^{e} & \leq\left(\sum_{i=1}^{m} a_{i}\right)^{e} \leq m^{e-1} \sum_{i=1}^{m} a_{i}^{e}, \quad 1 \leq e<\infty .
\end{aligned}
$$

The left inequalities can be proved by rescaling the $a_{i}$ by $\left(\sum_{i=1}^{m} a_{i}\right)^{-1}$ and using that for $a \in[0,1], a^{e} \leq a \Leftrightarrow e \geq 1$. The right inequalities are corollaries of Hölder's inequality: for $1<e<\infty$ and $a_{1}, \ldots, a_{m}, b_{1}, \ldots, b_{m} \geq 0$

$$
\sum_{i=1}^{m} a_{i} b_{i} \leq\left(\sum_{i=1}^{m} a_{i}^{e}\right)^{\frac{1}{e}}\left(\sum_{i=1}^{m} b_{i}^{\frac{e}{e-1}}\right)^{\frac{e-1}{e}} .
$$

2.6 Some properties of analytic functions

The following material will be used only in Section 6. For more details see, for example, 31.

Definition 1 A function $f: U \subseteq \mathbb{C}^{n} \rightarrow \mathbb{C}$ or $f: U \subseteq \mathbb{R}^{n} \rightarrow \mathbb{R}$ is analytic at $\boldsymbol{x}$ if there is an open neighborhood $\bar{U}_{x}$ of $\boldsymbol{x}$ where the sequence

$$
T_{d} f(\boldsymbol{y}):=\sum_{\boldsymbol{\alpha} \leq d} \frac{(\boldsymbol{y}-\boldsymbol{x})^{\boldsymbol{\alpha}}}{\boldsymbol{\alpha} !} \partial^{\boldsymbol{\alpha}} f(\boldsymbol{x})
$$

converges uniformly to $f$ as $d \rightarrow \infty$. A function is said to be analytic on $U$ if it is analytic at $\boldsymbol{x}$ for all $\boldsymbol{x} \in U$.

If $f: U \subset \mathbb{C} \rightarrow \mathbb{C}$ is analytic on $D_{x, r}:=\{z \in \mathbb{C}:|x-z| \leq r\}$ then the Cauchy formula states

$$
f(x)=\frac{1}{2 \pi i} \int_{\partial D_{x, r}} \frac{f(z)}{z-x} d z .
$$

This has a multivariate analogue [31, Theorem1.3] that follows by applying the above formula to each coordinate. Given $\boldsymbol{x} \in \mathbb{C}^{n}$ and $\mathbf{0}<\boldsymbol{r} \in \mathbb{R}^{n}$ let

$$
D_{\boldsymbol{x}, \boldsymbol{r}}:=D_{x_{1}, r_{1}} \times \cdots \times D_{x_{n}, r_{n}}, \quad \tilde{\partial} D_{\boldsymbol{x}, \boldsymbol{r}}:=\partial D_{x_{1}, r_{1}} \times \cdots \times \partial D_{x_{n}, r_{n}} .
$$

For $f: U \subset \mathbb{C}^{n} \rightarrow \mathbb{C}$ analytic on $D_{\boldsymbol{x}, \boldsymbol{r}}$ we have

$$
f(\boldsymbol{x})=(2 \pi i)^{-n} \int_{\tilde{\partial} D_{\boldsymbol{x}, \boldsymbol{r}}} \frac{f(\boldsymbol{z})}{(\boldsymbol{z}-\boldsymbol{x})^{\mathbf{1}}} d \boldsymbol{z} .
$$

The Cauchy formula implies the following proposition, see also [31, Theorem 1.6]. 
Proposition 1 Let $\Omega \subset \mathbb{C}^{n}$ be any set. If $f$ is analytic on $\Delta \subseteq \mathbb{C}^{n}$ containing $\Omega$ and

$$
\boldsymbol{r}(\boldsymbol{x}):=\max \left\{\boldsymbol{w}: D_{\boldsymbol{x}, \boldsymbol{w}} \subseteq \Delta\right\}>\mathbf{0}, \quad \forall \boldsymbol{x} \in \Omega
$$

then for all $\boldsymbol{x} \in \Omega$

$$
\left|\partial^{\boldsymbol{\alpha}} f(\boldsymbol{x})\right| \leq \frac{\boldsymbol{\alpha} !}{\boldsymbol{r}(\boldsymbol{x})^{\boldsymbol{\alpha}}}\|f\|_{\infty, \Delta}
$$

Proof Differentiating the Cauchy formula $\boldsymbol{\alpha}$ times with respect to $\boldsymbol{x}$ we have

$\partial^{\boldsymbol{\alpha}} f(\boldsymbol{x})=\partial^{\boldsymbol{\alpha}}(2 \pi i)^{-n} \int_{\tilde{\partial} D_{\boldsymbol{x}, \boldsymbol{r}(\boldsymbol{x})}} \frac{f(\boldsymbol{z})}{(\boldsymbol{z}-\boldsymbol{x})^{\mathbf{1}}} d \boldsymbol{z}=(2 \pi i)^{-n} \boldsymbol{\alpha} ! \int_{\tilde{\partial} D_{\boldsymbol{x}, \boldsymbol{r}(\boldsymbol{x})}} \frac{f(\boldsymbol{z})}{(\boldsymbol{z}-\boldsymbol{x})^{\boldsymbol{\alpha}+\mathbf{1}}} d \boldsymbol{z}$

and consequently

$$
\left|\partial^{\boldsymbol{\alpha}} f(\boldsymbol{x})\right| \leq(2 \pi)^{-n} \boldsymbol{\alpha} !\|f\|_{\infty, \Delta} \int_{\tilde{\partial} D_{\boldsymbol{x}, \boldsymbol{r}(\boldsymbol{x})}}\left|(\boldsymbol{z}-\boldsymbol{x})^{-\boldsymbol{\alpha}-\mathbf{1}}\right| d \boldsymbol{z}
$$

Since the integral equals $\boldsymbol{r}(\boldsymbol{x})^{-\boldsymbol{\alpha}}(2 \pi)^{n}$, the result follows.

Proposition 1 does not apply to real analytic function $U \subset \mathbb{R}^{n} \rightarrow \mathbb{R}$ and we use the following proposition:

Proposition 2 All real analytic functions $f: \Omega \subset \mathbb{R}^{n} \rightarrow \mathbb{R}$ admit an analytic extension to a neighborhood $\Delta \subset \mathbb{C}^{n}$ containing $\Omega$ and such that $\boldsymbol{r}(\boldsymbol{x})$ defined in (13) is strictly positive on $\Omega$.

Proof For $\boldsymbol{x} \in \Omega$, let $r(\boldsymbol{x})>0$ be the radius of convergence of the Taylor series of $f$ around $\boldsymbol{x}$. The series is absolutely convergent on $\left\{\boldsymbol{y} \in \mathbb{R}^{n}:\|\boldsymbol{x}-\boldsymbol{y}\|<r(\boldsymbol{x})\right\}$ and thus on $\stackrel{\circ}{D}_{\boldsymbol{x}, r(\boldsymbol{x})}:=\left\{\boldsymbol{y} \in \mathbb{C}^{n}:\|\boldsymbol{x}-\boldsymbol{y}\|<r(\boldsymbol{x})\right\}$. This implies that $f$ admits an analytic continuation to

$$
\Delta:=\bigcup_{\boldsymbol{x} \in \Omega} D_{\boldsymbol{x}, r(\boldsymbol{x}) / 2} \subset \bigcup_{\boldsymbol{x} \in \Omega} \stackrel{\circ}{D}_{\boldsymbol{x}, r(\boldsymbol{x})}
$$

and $\min \boldsymbol{r}(\boldsymbol{x}) \geq r(\boldsymbol{x}) / \sqrt{n}>0$ for all $\boldsymbol{x} \in \Omega$.

\section{Estimates on box meshes}

This section describes how error estimates of the form (1) follow from the assumptions $\mathrm{H}_{\mathbb{P}} \ldots \mathrm{H}_{[\mathrm{s}}$. In the first subsection local error estimates are derived from $\mathrm{H}_{\mathbb{P}} \ldots \mathrm{H}_{\Pi}$. Global error bounds require additionally $\mathrm{H}_{\mathrm{E}} \ldots \mathrm{H}_{\mathrm{S}}$ and are stated in the second and third subsections. The fourth subsection describes the dependence of the mesh assumption on the parameters of $\mathrm{H}_{\Pi}$

It is important to highlight that error estimates can only be obtained for suitable choices of the Sobolev spaces involved and this will be discussed in Section 4 where $\mathrm{H}_{\Pi}$ is shown for the averaged Taylor expansion operator. 


\subsection{Local error}

Usually local error bounds are stated using the support extension

$$
\|f-\aleph f\|_{p, \omega} \leq C \inf _{g \in \mathbb{P}}\|f-g\|_{p, \operatorname{ext} \omega}, \quad \operatorname{ext} \omega:=\bigcup_{\phi \in \mathrm{A}_{\omega}} \operatorname{supp} \lambda_{\phi}
$$

The drawback of this approach is that the polynomial approximation properties depends on the shape of ext $\omega$. Common assumptions for polynomial approximation are that the domain is star-shaped with respect to a subset of positive measure [17] [18 41] or bounded by graphs of regular functions [32. In 39] local estimates for the approximation error are obtained without shape constraints on ext $\omega$ by using the convex hull of ext $\omega$. Unfortunately this technique cannot be extended to global estimates because it is difficult to bound the number of overlapping hulls in terms of elementary properties of the mesh.

Here we avoid the support extension by using the form

$$
\|f-\aleph f\|_{p, \omega} \leq C \inf _{g \in \mathbb{P}} \sum_{\phi \in \mathrm{A}_{\omega}}\|f-g\|_{p, \mathbf{s}_{\phi}} .
$$

This reduces the complexity of the shape on which we use polynomial approximation and allow us to derive global estimates and to reduce the mesh regularity assumptions.

The local error bounds are based on the following assumptions ${ }^{1}$ where we use $\boldsymbol{h}_{\phi}$ as a shortening for $\boldsymbol{h}_{\operatorname{supp} \phi}$.

$\mathrm{H}_{\mathbb{P}} \aleph g=g$ for all $g \in \mathbb{P}_{\boldsymbol{d}}$, polynomial reproduction;

$\mathrm{H}_{\lambda}\left\|\lambda_{\phi}\right\|_{* p}\|\phi\|_{p} \leq C_{\lambda}$, for all $\phi \in \Phi$, functional continuity;

$\mathrm{H}_{\phi} \partial^{\sigma} \phi \in L^{p}(\Omega)$, for all $\phi \in \Phi$, and $\left\|\partial^{\boldsymbol{\sigma}} \phi\right\|_{p, \omega} \leq C_{\Phi} \boldsymbol{h}_{\phi}^{-\boldsymbol{\sigma}} \frac{\mu(\omega)^{\frac{1}{p}}}{\mu(\operatorname{supp} \phi)^{\frac{1}{p}}}\|\phi\|_{p}$, generators' regularity;

$\mathrm{H}_{\mathbf{s}} \boldsymbol{h}_{\mathbf{s}_{\phi}} \leq C_{\mathbf{s}} \boldsymbol{h}_{\phi}$, and $\boldsymbol{h}_{\mathbf{s}_{\phi}}^{\mathbf{1}} \leq C_{\mathbf{s}}^{n} \mu(\operatorname{supp} \phi)$ for all $\phi \in \Phi$, locality of $\aleph ;$

$\mathrm{H}_{\Pi}$ for each $\omega \in \mathcal{M}$ there is an approximation operator $\Pi_{\omega}: L^{p}(\omega) \rightarrow \mathbb{P}_{\boldsymbol{d}}$ such that for $\boldsymbol{\beta} \in\{\mathbf{0}, \boldsymbol{\sigma}\}, \eta \in\{\omega\} \cup\left\{\mathbf{s}_{\phi}: \phi \in \mathrm{A}_{\omega}\right\}$ it holds

$$
\left\|\partial^{\boldsymbol{\beta}}\left(f-\Pi_{\omega} f\right)\right\|_{p, \eta} \leq C_{\Pi} \frac{\boldsymbol{h}_{\omega}^{\gamma}}{\boldsymbol{h}_{\eta}^{\gamma}} \sum_{\boldsymbol{k} \in K_{\boldsymbol{\beta}}} \boldsymbol{h}_{\eta}^{\boldsymbol{k}-\boldsymbol{\beta}+\boldsymbol{\nu}}\left\|\partial^{\boldsymbol{k}} f\right\|_{q, \eta}, \quad \boldsymbol{\nu}=\mathbf{1} / p-\mathbf{1} / q .
$$

Note that $C_{\mathrm{s}} \geq 1$, and for simplicity we assume that also $C_{\lambda}, C_{\Phi} \geq 1$.

The abstract upper bound for polynomial approximation in $\mathrm{H}_{\Pi}$ depends on the index sets $K_{\mathbf{0}}, K_{\boldsymbol{\sigma}} \subset \mathbb{N}^{n}$ and the parameter $\gamma \in \mathbb{R}^{n}$ that influences the required mesh assumptions. In Section 5 we describe operators $\Pi_{\omega}$ leading to error bounds with Sobolev and reduced seminorms.

Remark 1 If $\operatorname{supp} \lambda_{\phi} \subseteq \operatorname{supp} \phi=\mathrm{b}\left(\mathbf{s}_{\phi}\right)$ then $\mathrm{H}_{\mathrm{s}}$ is satisfied with $C_{\mathrm{s}}=1$. This will be the case for the proposed operators for TPS, LR and AST splines. The control on the support measure in $\mathrm{H}_{\mathbf{s}}$ is needed if $\operatorname{supp} \phi$ or $\mathbf{s}_{\phi}$ is not a box. This happens for THB and non-convex domains.

\footnotetext{
1 All the assumptions are conveniently listed in the last page, see Table 1
} 
Theorem 1 (Local error) For $1 \leq p, q \leq \infty$ and assuming $\mathrm{H}_{\mathbb{P}} \ldots \mathrm{H}_{\Pi}$ we have

$$
\begin{aligned}
& \left\|\partial^{\boldsymbol{\sigma}}(f-\aleph f)\right\|_{p, \omega} \leq C_{\lambda} C_{\Phi} C_{\mathbf{s}}^{|\boldsymbol{\sigma}|+\frac{n}{p}} C_{\Pi} \\
& \quad\left[\sum_{\boldsymbol{k} \in K_{\boldsymbol{\sigma}}} \boldsymbol{h}_{\omega}^{\boldsymbol{k}-\boldsymbol{\sigma}+\boldsymbol{\nu}}\left\|\partial^{\boldsymbol{k}} f\right\|_{q, \omega}+\sum_{\phi \in \mathrm{A}_{\omega}} \frac{\boldsymbol{h}_{\omega}^{\gamma+\mathbf{1} / p}}{\boldsymbol{h}_{\mathbf{s}_{\phi}}^{\gamma+\mathbf{1} / p}} \sum_{\boldsymbol{k} \in K_{\mathbf{0}}} \boldsymbol{h}_{\mathbf{s}_{\phi}}^{\boldsymbol{k}-\boldsymbol{\sigma}+\boldsymbol{\nu}}\left\|\partial^{\boldsymbol{k}} f\right\|_{q, \mathbf{s}_{\phi}}\right] .
\end{aligned}
$$

Moreover, if $\boldsymbol{\sigma}=\mathbf{0}$ and $p=\infty$ the same inequality holds under the reduced assumptions $\mathrm{H}_{\mathbb{P}}, \mathrm{H}_{\lambda} \mathrm{H}_{\phi}$ and $\mathrm{H}_{\Pi}$.

Proof The reproduction of polynomial property $\mathrm{H}_{\mathbb{P}}$ and linearity of $\aleph$ imply that for all $g \in \mathbb{P}_{\boldsymbol{d}}$

$$
\begin{aligned}
\left\|\partial^{\sigma}(f-\aleph f)\right\|_{p, \omega} & =\left\|\partial^{\sigma}(f-g)-\partial^{\sigma} \aleph(f-g)\right\|_{p, \omega} \\
& =\left\|\partial^{\sigma}(f-g)-\sum_{\phi \in \mathrm{A}_{\omega}} \lambda_{\phi}(f-g) \partial^{\sigma} \phi\right\|_{p, \omega} \\
& \leq\left\|\partial^{\sigma}(f-g)\right\|_{p, \omega}+\sum_{\phi \in \mathrm{A}_{\omega}}\left\|\partial^{\sigma} \phi\right\|_{p, \omega}\left|\lambda_{\phi}(f-g)\right| .
\end{aligned}
$$

By $\mathrm{H}_{\mathrm{s}}$ we obtain

$$
\boldsymbol{h}_{\phi}^{-\boldsymbol{\sigma}} \leq C_{\mathbf{s}}^{|\boldsymbol{\sigma}|} \boldsymbol{h}_{\mathbf{s}_{\phi}}^{-\boldsymbol{\sigma}}, \quad \mu(\omega)^{1 / p}=\boldsymbol{h}_{\omega}^{\mathbf{1} / p}, \quad \mu(\operatorname{supp} \phi)^{-1 / p} \leq C_{\mathbf{s}}^{n / p} \boldsymbol{h}_{\mathbf{s}_{\phi}}^{-\mathbf{1} / p} .
$$

Using this, $\mathrm{H}_{\phi}, \mathrm{H}_{\lambda}$ and $\mathrm{H}_{\Pi}$ with $g=\Pi_{\omega} f$ we have

$$
\begin{gathered}
\left\|\partial^{\boldsymbol{\sigma}} \phi\right\|_{p, \omega}\left|\lambda_{\phi}(f-g)\right| \leq C_{\Phi} \boldsymbol{h}_{\mathbf{s}_{\phi}}^{-\boldsymbol{\sigma}} \frac{\mu(w)^{1 / p}}{\mu(\operatorname{supp} \phi)^{1 / p}}\|\phi\|_{p}\left\|\lambda_{\phi}\right\|_{* p}\|f-g\|_{p, \operatorname{supp} \lambda_{\phi}} \\
\leq C_{\Phi} C_{\mathbf{s}}^{|\boldsymbol{\sigma}|} \boldsymbol{h}_{\mathbf{s}_{\phi}}^{-\boldsymbol{\sigma}} \boldsymbol{h}_{\omega}^{\mathbf{1} / p} C_{\mathbf{s}}^{n / p} \boldsymbol{h}_{\mathbf{s}_{\phi}}^{-\mathbf{1} / p} C_{\lambda} C_{\Pi} \boldsymbol{h}_{\omega}^{\gamma} \boldsymbol{h}_{\mathbf{s}_{\phi}}^{-\gamma} \sum_{\boldsymbol{k} \in K_{\mathbf{0}}} \boldsymbol{h}_{\mathbf{s}_{\phi}}^{\boldsymbol{k}+\boldsymbol{\nu}}\left\|\partial^{\boldsymbol{k}} f\right\|_{q, \mathbf{s}_{\phi}}
\end{gathered}
$$

Summing over the $\phi \in \mathrm{A}_{\omega}$ and simplifying we obtain the second term in 15 . Using $\mathrm{H}_{\Pi}$ with $\eta=\omega$ and $\boldsymbol{\beta}=\boldsymbol{\sigma}$ we have

$$
\left\|\partial^{\sigma}\left(f-\Pi_{\omega} f\right)\right\|_{p, \omega} \leq C_{\Pi} \sum_{\boldsymbol{k} \in K_{\sigma}} \boldsymbol{h}_{\omega}^{\boldsymbol{k}-\boldsymbol{\sigma}+\boldsymbol{\nu}}\left\|\partial^{\boldsymbol{k}} f\right\|_{q, \omega}
$$

and 15 follows since $C_{\lambda} C_{\Phi} C_{\mathrm{s}}^{|\sigma|+\frac{n}{p}} \geq 1$.

\subsection{Global error $p<\infty$}

The global error bound is obtained by summing the local bounds from Theorem 1 . The procedure uses the following additional assumptions

$\mathrm{H}_{\mathrm{E}} \# \mathrm{E}_{\omega} \leq C_{\mathrm{E}}$, for all $\omega \in \mathcal{M}$, bound on $\mathbf{s}_{\phi}$ overlaps;

$\mathrm{H}_{\mathcal{M}} \Gamma_{\phi} \leq C_{\mathcal{M}}$ for all $\phi \in \Phi$, mesh regularity, where

$$
\Gamma_{\phi}:= \begin{cases}\left(\sum_{\omega \in \mathbb{M}_{\phi}} \frac{\boldsymbol{h}_{\omega}^{\gamma p+1}}{\boldsymbol{h}_{\mathbf{s}_{\phi}}^{\gamma p+1}}\right)^{\frac{1}{p}} & p \neq \infty \\ \max _{\omega \in \mathbb{M}_{\phi}}\left\{\frac{\boldsymbol{h}_{\omega}^{\gamma}}{\boldsymbol{h}_{\mathbf{s}_{\phi}}^{\gamma}}\right\} & p=\infty,\end{cases}
$$

and $\gamma$ was introduced in $\mathrm{H}_{\Pi}$ 
$\mathrm{H}_{\varpi} \max \boldsymbol{h}_{\omega} \leq C_{\varpi} \min \boldsymbol{h}_{\omega}$ for all $\omega \in \mathcal{M}$, element shape regularity;

$\mathrm{H}_{[\mathbf{s}]} \max \boldsymbol{h}_{\mathbf{s}_{\phi}} \leq C_{\mathbf{S} \mathbf{s}} \min \boldsymbol{h}_{\mathbf{s}_{\phi}}$ for all $\phi \in \Phi$, shape regularity of $\mathbf{s}_{\phi}$.

Note that $C_{\mathrm{E}}, C_{\omega}, C_{\mathbf{S}} \geq 1$, and for simplicity we assume that also $C_{\mathcal{M}} \geq 1$.

$\mathrm{H}_{\mathcal{M}}$ is a mesh regularity property that mirrors the result of the computations. It is implied by the usual assumptions for non-tensor-product spaces, but it allows for weaker mesh regularity if $\gamma \geq \mathbf{- 1 / p}$. In particular tiny and not shape regular elements are allowed as long as each extended support is shape regular and contains a bounded number of elements, see Subsection 3.4 . Assumptions $\mathrm{H}_{[\boldsymbol{\omega}}$ and $\mathrm{H}_{\mathbf{S}}$ will be used only if $\boldsymbol{\sigma} \neq 0$ or if $p>q$.

Remark 2 As $\mathrm{E}_{\omega} \supseteq \mathrm{A}_{\omega}$, we have $\# \mathrm{~A}_{\omega} \leq \# \mathrm{E}_{\omega} \leq C_{\mathrm{E}}$. Some estimates can be made slightly sharper by using an upper bound for $\# \mathrm{~A}_{\omega}$.

Theorem 2 (Global error) For $1 \leq p<\infty, 1 \leq q \leq \infty$ and assuming $\mathrm{H}_{\mathbb{P}}, \ldots, \mathrm{H}_{M}$ we have

$$
\left\|\partial^{\sigma}(f-\aleph f)\right\|_{p} \leq C \mu(\Omega)^{\nu_{+}}\left(\sum_{\boldsymbol{k} \in K_{\boldsymbol{\sigma}}}\left\|\mathrm{h}_{\mathcal{M}}^{\boldsymbol{k}-\boldsymbol{\sigma}+\boldsymbol{\nu}_{-}} \partial^{\boldsymbol{k}} f\right\|_{q}+\sum_{\boldsymbol{k} \in K_{\mathbf{0}}}\left\|\rho_{\boldsymbol{k}, \boldsymbol{\sigma}} \partial^{\boldsymbol{k}} f\right\|_{q}\right),
$$

where we recall that $\boldsymbol{\nu}:=\mathbf{1} / p-\mathbf{1} / q, \mathrm{~h}_{\mathcal{M}}$ is defined in $(9), \rho_{\boldsymbol{k}, \boldsymbol{\sigma}}: \Omega \rightarrow \mathbb{R}$ is the piecewise constant function defined by $\left.\rho_{\boldsymbol{k}, \boldsymbol{\sigma}}\right|_{\omega}:=\max _{\phi \in \mathrm{E}_{\omega}}\left\{\boldsymbol{h}_{\mathbf{s}_{\phi}}^{\boldsymbol{k}-\boldsymbol{\sigma}+\boldsymbol{\nu}_{-}}\right\}$and

$$
C:=2^{1-\frac{1}{p}} \#\left(K_{\boldsymbol{\sigma}} \cup K_{\mathbf{0}}\right)^{1-1 / p} C_{\lambda} C_{\Phi} C_{\mathrm{s}}^{|\boldsymbol{\sigma}|+\left|\boldsymbol{\nu}_{+}\right|+\frac{n}{p}} C_{\Pi} C_{\mathrm{E}}^{1-\nu_{-}} C_{\mathcal{M}}
$$

Before giving the proof we state two Corollaries.

Corollary 1 For $1 \leq p \leq q \leq \infty, p \neq \infty$ and assuming $\overline{\mathrm{H}_{\mathbb{P}}}$. . $\mathrm{H}_{\mathcal{M}}$ we have

$$
\|f-\aleph f\|_{p} \leq C \mu(\Omega)^{\nu_{+}} \sum_{\boldsymbol{k} \in K_{\mathbf{0}}}\left\|\mathrm{h}_{\Phi}^{\boldsymbol{k}} \partial^{\boldsymbol{k}} f\right\|_{q}
$$

where $C:=4 \# K_{\mathbf{0}}{ }^{1-\frac{1}{p}} C_{\lambda} C_{\Phi} C_{\mathrm{s}}^{n\left(\frac{2}{p}-\frac{1}{q}\right)} C_{\Pi} C_{\mathrm{E}} C_{\mathcal{M}}$.

Proof Since $\left.\mathrm{h}_{\mathcal{M}}\right|_{\omega}=\boldsymbol{h}_{\omega} \leq \max _{\phi \in \mathrm{E}_{\omega}} \boldsymbol{h}_{\mathrm{s}_{\phi}}=\left.\mathrm{h}_{\Phi}\right|_{\omega}$ and $K_{\mathbf{0}} \geq \mathbf{0}$ we have $\mathrm{h}_{\mathcal{M}}^{\boldsymbol{k}} \leq \mathrm{h}_{\Phi}^{\boldsymbol{k}}$. Moreover, $\boldsymbol{\nu}_{-}=\mathbf{0}$ because $p \leq q$, so that

$$
\left.\rho_{\boldsymbol{k}, \mathbf{0}}\right|_{\omega}=\max _{\phi \in \mathrm{E}_{\omega}} \prod_{i=1}^{n} h_{\mathbf{s}_{\phi}, i}^{k_{i}} \leq \prod_{i=1}^{n} \max _{\phi \in \mathrm{E}_{\omega}} h_{\mathbf{s}_{\phi}, i}^{k_{i}}=\left.\mathbf{h}_{\Phi}^{\boldsymbol{k}}\right|_{\omega} .
$$

Inserting the above in Theorem 2 gives the result.

Corollary 2 For $1 \leq p, q \leq \infty, p \neq \infty$ and assuming $\mathrm{H}_{\mathbb{P}} \ldots, \mathrm{H}_{\mathbb{S}}, K_{\boldsymbol{\sigma}} \geq \boldsymbol{\sigma}$ and $|\boldsymbol{k}| \geq|\boldsymbol{\sigma}|+\left|\boldsymbol{\nu}_{-}\right|$for $\boldsymbol{k} \in K_{\mathbf{0}} \cup K_{\boldsymbol{\sigma}}$, we have

$$
\begin{aligned}
\left\|\partial^{\boldsymbol{\sigma}}(f-\aleph f)\right\|_{p} \leq & C\left(C_{[}^{\left|\boldsymbol{\nu}_{-}\right|}+C_{[\mathbf{S}]}^{|\boldsymbol{\sigma}|+\left|\boldsymbol{\nu}_{-}\right|}\right) \\
& \mu(\Omega)^{\nu_{+}} \sum_{\boldsymbol{k} \in K_{\boldsymbol{\sigma}} \cup K_{\mathbf{0}}}\left\|\left(\operatorname{maxh}_{\Phi}\right)^{|\boldsymbol{k}|-|\boldsymbol{\sigma}|-\left|\boldsymbol{\nu}_{-}\right|} \partial^{\boldsymbol{k}} f\right\|_{q},
\end{aligned}
$$

where $C$ is given in Theorem 2. Moreover, if $p \leq q$ then $\mathrm{H}_{\text {G }}$ is not required. If $p \leq q$ and $\boldsymbol{\sigma}=\mathbf{0}$ then $\overline{\mathrm{H}_{[\omega]}}$ and $\overline{\mathrm{H}_{[\mathbf{S}}}$ are not required. 
Proof With $\boldsymbol{\alpha}=\boldsymbol{k}-\boldsymbol{\sigma}+\boldsymbol{\nu}_{-}$and using (4) and $\mathrm{H}_{\mathbf{\omega}}$ we have

$$
\left.\mathrm{h}_{\mathcal{M}}^{\boldsymbol{\alpha}}\right|_{\omega} \leq\left(\max \boldsymbol{h}_{\omega}\right)^{\left|\boldsymbol{\alpha}_{+}\right|}\left(\min \boldsymbol{h}_{\omega}\right)^{-\left|\boldsymbol{\alpha}_{-}\right|} \leq C_{\varpi}^{\left|\boldsymbol{\alpha}_{-}\right|}\left(\max \boldsymbol{h}_{\omega}\right)^{\left|\boldsymbol{\alpha}_{+}\right|-\left|\boldsymbol{\alpha}_{-}\right|} .
$$

Since $\boldsymbol{k} \geq \boldsymbol{\sigma}$ we have $\left|\boldsymbol{\nu}_{-}\right| \geq\left|\boldsymbol{\alpha}_{-}\right|$and using $C_{\varpi} \geq 1$ and $\max \boldsymbol{h}_{\omega} \leq\left.\operatorname{maxh}_{\Phi}\right|_{\omega}$ we obtain

$$
\mathrm{h}_{\mathcal{M}}^{\boldsymbol{k}-\boldsymbol{\sigma}+\boldsymbol{\nu}_{-}} \leq C_{\varpi}^{\left|\boldsymbol{\nu}_{-}\right|}\left(\operatorname{maxh}_{\Phi}\right)^{|\boldsymbol{k}|-|\boldsymbol{\sigma}|-\left|\boldsymbol{\nu}_{-}\right|} .
$$

Note that for $p \leq q$ and $\boldsymbol{k} \in K_{\boldsymbol{\sigma}}$ then $\boldsymbol{\alpha} \geq \mathbf{0}$ and $\mathrm{H}_{\omega}$ is not required. Similarly using $\mathrm{H}_{[\mathbf{S}}$ and $\left|\boldsymbol{\alpha}_{-}\right| \leq|\boldsymbol{\sigma}|+\left|\boldsymbol{\nu}_{-}\right|$we obtain

$$
\rho_{\boldsymbol{k}, \boldsymbol{\sigma}} \leq C_{[\mathbf{S}}^{|\boldsymbol{\sigma}|+\left|\boldsymbol{\nu}_{-}\right|}\left(\operatorname{maxh}_{\Phi}\right)^{|\boldsymbol{k}|-|\boldsymbol{\sigma}|-\left|\boldsymbol{\nu}_{-}\right|} .
$$

Moreover, for $p \leq q$ and $\boldsymbol{\sigma}=\mathbf{0}$ we have $K_{\mathbf{0}}=K_{\boldsymbol{\sigma}} \geq \mathbf{0}$ and $\mathrm{H}_{\mathbf{s}}$ is not required. Inserting (24) and 25) in Theorem 2 gives the result.

Proof (Theorem 2) From $\left\|\partial^{\sigma}(f-\aleph f)\right\|_{p}=\left(\sum_{\omega \in \mathcal{M}}\left\|\partial^{\sigma}(f-\aleph f)\right\|_{p, \omega}^{p}\right)^{1 / p}$, 15 and (10) with $e=p \geq 1$ and $m=\# K_{\boldsymbol{\sigma}}+\# \mathrm{~A}_{\omega} \# K_{\mathbf{0}} \leq 2 \#\left(K_{\boldsymbol{\sigma}} \cup K_{\mathbf{0}}\right) C_{\mathrm{E}}$ and again (10) with $e=1 / p \leq 1$ we get

$$
\begin{gathered}
\left\|\partial^{\boldsymbol{\sigma}}(f-\aleph f)\right\|_{p} \leq 2^{1-\frac{1}{p}} \#\left(K_{\boldsymbol{\sigma}} \cup K_{\mathbf{0}}\right)^{1-\frac{1}{p}} C_{\lambda} C_{\Phi} C_{\mathbf{s}}^{|\boldsymbol{\sigma}|+n / p} C_{\Pi} C_{\mathrm{E}}^{1-\frac{1}{p}} \\
{\left[\sum_{\boldsymbol{k} \in K_{\boldsymbol{\sigma}}}\left(\sum_{\omega \in \mathcal{M}} \boldsymbol{h}_{\omega}^{p(\boldsymbol{k}-\boldsymbol{\sigma}+\boldsymbol{\nu})}\left\|\partial^{\boldsymbol{k}} f\right\|_{q, \omega}^{p}\right)^{\frac{1}{p}}\right.} \\
\left.\quad+\sum_{\boldsymbol{k} \in K_{\mathbf{0}}}\left(\sum_{\omega \in \mathcal{M}} \sum_{\phi \in \mathrm{A}_{\omega}} \frac{\boldsymbol{h}_{\omega}^{\gamma p+\mathbf{1}}}{\boldsymbol{h}_{\mathbf{s}_{\phi}}^{\gamma p+\mathbf{1}}} \boldsymbol{h}_{\mathbf{s}_{\phi}^{p(\boldsymbol{k}-\boldsymbol{\sigma}+\boldsymbol{\nu})}\left\|\partial^{\boldsymbol{k}} f\right\|_{q, \mathbf{s}_{\phi}}^{p}}\right)^{\frac{1}{p}}\right] .
\end{gathered}
$$

By changing the summation order we have

$$
\sum_{\omega \in \mathcal{M}} \sum_{\phi \in \mathrm{A}_{\omega}} \frac{\boldsymbol{h}_{\omega}^{\gamma p+\mathbf{1}}}{\boldsymbol{h}_{\mathbf{s}_{\phi}}^{\gamma p+\mathbf{1}}} \boldsymbol{h}_{\mathbf{s}_{\phi}}^{p(\boldsymbol{k}-\boldsymbol{\sigma}+\boldsymbol{\nu})}\left\|\partial^{\boldsymbol{k}} f\right\|_{q, \mathbf{s}_{\phi}}^{p}=\sum_{\phi \in \Phi} \sum_{\omega \in \mathbb{M}_{\phi}} \frac{\boldsymbol{h}_{\omega}^{\gamma p+\mathbf{1}}}{\boldsymbol{h}_{\mathbf{s}_{\phi}}^{\gamma p+\mathbf{1}}} \boldsymbol{h}_{\mathbf{s}_{\phi}}^{p(\boldsymbol{k}-\boldsymbol{\sigma}+\boldsymbol{\nu})}\left\|\partial^{\boldsymbol{k}} f\right\|_{q, \mathbf{s}_{\phi}}^{p} .
$$

Using $\mathrm{H}_{\mathcal{M}}$ this leads to

$$
\begin{aligned}
& \left\|\partial^{\boldsymbol{\sigma}}(f-\aleph f)\right\|_{p} \leq 2^{1-\frac{1}{p}} \#\left(K_{\boldsymbol{\sigma}} \cup K_{\mathbf{0}}\right)^{1-\frac{1}{p}} C_{\lambda} C_{\Phi} C_{\mathrm{s}}^{|\boldsymbol{\sigma}|+\frac{n}{p}} C_{\Pi} C_{\mathrm{E}}^{1-\frac{1}{p}} C_{\mathcal{M}} \\
& {\left[\sum_{\boldsymbol{k} \in K_{\boldsymbol{\sigma}}}\left(\sum_{\omega \in \mathcal{M}} \boldsymbol{h}_{\omega}^{p(\boldsymbol{k}-\boldsymbol{\sigma}+\boldsymbol{\nu})}\left\|\partial^{\boldsymbol{k}} f\right\|_{q, \omega}^{p}\right)^{\frac{1}{p}}+\sum_{\boldsymbol{k} \in K_{\mathbf{0}}}\left(\sum_{\phi \in \Phi} \boldsymbol{h}_{\mathbf{s}_{\phi}}^{p(\boldsymbol{k}-\boldsymbol{\sigma}+\boldsymbol{\nu})}\left\|\partial^{\boldsymbol{k}} f\right\|_{q, \mathbf{s}_{\phi}}^{p}\right)^{\frac{1}{p}}\right] .}
\end{aligned}
$$

For $p \leq q$, Hölder inequality 11 with $a_{i}=\boldsymbol{h}_{\omega}^{p \nu}$ and $e=(p \nu)^{-1} \geq 1$ implies

$$
\begin{aligned}
\sum_{\omega \in \mathcal{M}} \boldsymbol{h}_{\omega}^{p(\boldsymbol{k}-\boldsymbol{\sigma}+\boldsymbol{\nu})}\left\|\partial^{\boldsymbol{k}} f\right\|_{q, \omega}^{p} & \leq\left(\sum_{\omega \in \mathcal{M}} \boldsymbol{h}_{\omega}^{\mathbf{1}}\right)^{p \nu}\left(\sum_{\omega \in \mathcal{M}} \boldsymbol{h}_{\omega}^{q(\boldsymbol{k}-\boldsymbol{\sigma})}\left\|\partial^{\boldsymbol{k}} f\right\|_{q, \omega}^{q}\right)^{\frac{p}{q}} \\
& \leq \mu(\Omega)^{p \nu}\left\|\mathbf{h}_{\mathcal{M}}^{\boldsymbol{k}-\boldsymbol{\sigma}} \partial^{\boldsymbol{k}} f\right\|_{q}^{p}
\end{aligned}
$$

Similarly we get

$$
\begin{aligned}
\sum_{\phi \in \Phi} \boldsymbol{h}_{\mathbf{s}_{\phi}}^{p(\boldsymbol{k}-\boldsymbol{\sigma}+\boldsymbol{\nu})}\left\|\partial^{\boldsymbol{k}} f\right\|_{q, \mathbf{s}_{\phi}}^{p} & \leq\left(\sum_{\phi \in \Phi} \boldsymbol{h}_{\mathbf{s}_{\phi}}^{\mathbf{1}}\right)^{p \nu}\left(\sum_{\phi \in \Phi} \boldsymbol{h}_{\mathbf{s}_{\phi}}^{q(\boldsymbol{k}-\boldsymbol{\sigma})}\left\|\partial^{\boldsymbol{k}} f\right\|_{q, \mathbf{s}_{\phi}}^{q}\right)^{\frac{p}{q}} \\
& \leq C_{\mathrm{E}} C_{\mathbf{s}}^{n p \nu} \mu(\Omega)^{p \nu}\left\|\rho_{\boldsymbol{k}, \boldsymbol{\sigma}} \partial^{\boldsymbol{k}} f\right\|_{q}^{p}
\end{aligned}
$$


where we used $\mathrm{H}_{\mathrm{s}}$ and $\mathrm{H}_{\mathrm{E}}$ to obtain

$$
\sum_{\phi \in \Phi} \boldsymbol{h}_{\mathbf{s}_{\phi}}^{1} \leq C_{\mathbf{s}}^{n} \sum_{\phi \in \Phi} \mu(\operatorname{supp} \phi) \leq C_{\mathbf{s}}^{n} C_{\mathrm{E}} \mu(\Omega),
$$

and the characteristic functions $\mathbb{1}_{\mathbf{s}_{\phi}}$ to obtain

$$
\begin{aligned}
\sum_{\phi \in \Phi} \boldsymbol{h}_{\mathbf{s}_{\phi}}^{q(\boldsymbol{k}-\boldsymbol{\sigma})}\left\|\partial^{\boldsymbol{k}} f\right\|_{q, \mathbf{s}_{\phi}}^{q} & =\int_{\Omega}\left(\sum_{\phi \in \Phi} \mathbb{1}_{\mathbf{s}_{\phi}}(\boldsymbol{x}) \boldsymbol{h}_{\mathbf{s}_{\phi}}^{q(\boldsymbol{k}-\boldsymbol{\sigma})}\right)\left|\partial^{\boldsymbol{k}} f(\boldsymbol{x})\right|^{q} d \boldsymbol{x} \\
& \leq \int_{\Omega} C_{\mathrm{E}} \rho_{\boldsymbol{k}, \boldsymbol{\sigma}}^{q}\left|\partial^{\boldsymbol{k}} f(\boldsymbol{x})\right|^{q} d \boldsymbol{x}=C_{\mathrm{E}}\left\|\rho_{\boldsymbol{k}, \boldsymbol{\sigma}} \partial^{\boldsymbol{k}} f\right\|_{q}^{q}
\end{aligned}
$$

Inserting (27) and (28) in (26) gives $(19)$ for $p \leq q$.

If $p>q$ then using (10) with $e=p / q \geq 1$ we have

$$
\begin{aligned}
\sum_{\omega \in \mathcal{M}} \boldsymbol{h}_{\omega}^{p(\boldsymbol{k}-\boldsymbol{\sigma}+\boldsymbol{\nu})}\left\|\partial^{k} f\right\|_{q, \omega}^{p} & \leq\left(\sum_{\omega \in \mathcal{M}} \boldsymbol{h}_{\omega}^{q(\boldsymbol{k}-\boldsymbol{\sigma}+\boldsymbol{\nu})}\left\|\partial^{k} f\right\|_{q, \omega}^{q}\right)^{\frac{p}{q}} \\
& =\left\|\mathrm{h}_{\mathcal{M}}^{\boldsymbol{k}-\boldsymbol{\sigma}+\boldsymbol{\nu}} \partial^{k} f\right\|_{q}^{p} .
\end{aligned}
$$

Similarly

$$
\begin{gathered}
\sum_{\phi \in \mathrm{A}_{\omega}} \boldsymbol{h}_{\mathbf{s}_{\phi}}^{p(\boldsymbol{k}-\boldsymbol{\sigma}+\boldsymbol{\nu})}\left\|\partial^{\boldsymbol{k}} f\right\|_{q, \mathbf{s}_{\phi}}^{p} \leq\left(\sum_{\phi \in \mathrm{A}_{\omega}} \boldsymbol{h}_{\mathbf{s}_{\phi}}^{q(\boldsymbol{k}-\boldsymbol{\sigma}+\boldsymbol{\nu})}\left\|\partial^{\boldsymbol{k}} f\right\|_{q, \mathbf{s}_{\phi}}^{q}\right)^{\frac{p}{q}} \\
=\left(\sum_{\phi \in \Phi} \int_{\Omega} \mathbb{1}_{\mathbf{s}_{\phi}}(\boldsymbol{x}) \boldsymbol{h}_{\mathbf{s}_{\phi}}^{q(\boldsymbol{k}-\boldsymbol{\sigma}+\nu)}\left|\partial^{\boldsymbol{k}} f(\boldsymbol{x})\right|^{q} d \boldsymbol{x}\right)^{\frac{p}{q}} \\
\leq C_{\mathrm{E}}^{\underline{p}}\left\|\rho_{\boldsymbol{k}, \boldsymbol{\sigma}} \partial^{\boldsymbol{k}} f\right\|_{q}^{p} .
\end{gathered}
$$

Inserting (29) and (30) in 26) gives (19) for $p \geq q$.

3.3 Global error $p=\infty$

For $p=\infty, \mathrm{H}_{\mathrm{E}}$ can be replaced with a bound on $\# \mathrm{~A}_{\omega}$, see Remark 2 Moreover, we have $\boldsymbol{\nu}=\boldsymbol{\nu}_{-}=-\mathbf{1} / q$.

Theorem 3 Assuming $\mathrm{H}_{\mathbb{P}}, \ldots, \mathrm{H}_{M}$, we have

$$
\|f-\aleph f\|_{\infty} \leq C \max _{\phi \in \Phi}\left\{\sum_{k \in K_{0}}\left\|\boldsymbol{h}_{\mathbf{s}_{\phi}}^{k} \partial^{k} f\right\|_{\infty, \mathbf{s}_{\phi}}\right\},
$$

where $C:=2 C_{\lambda} C_{\Phi} C_{\Pi} C_{\mathbf{s}}^{|\boldsymbol{\sigma}|} C_{\mathrm{E}} C_{\mathcal{M}}$. Assuming in addition $\mathrm{H}_{\omega}, \mathrm{H}_{\mathbf{s}}, K_{\boldsymbol{\sigma}} \geq \boldsymbol{\sigma}$ and $|\boldsymbol{k}| \geq|\boldsymbol{\sigma}|+\left|\boldsymbol{\nu}_{-}\right|$for $\boldsymbol{k} \in K_{\mathbf{0}} \cup K_{\boldsymbol{\sigma}}$ we have with the same $C$

$$
\begin{aligned}
\left\|\partial^{\boldsymbol{\sigma}}(f-\aleph f)\right\|_{\infty} \leq C\left(C_{[\underline{\omega}}^{|\boldsymbol{\nu}|}+C_{\mathbf{[}]}^{|\boldsymbol{\sigma}|+\left|\boldsymbol{\nu}_{-}\right|}\right) & \max _{\phi \in \Phi}\left\{\sum_{\boldsymbol{k} \in K_{\sigma} \cup K_{\mathbf{0}}}\left\|\left(\max \boldsymbol{h}_{\mathbf{s}_{\phi}}\right)^{|\boldsymbol{k}|-|\boldsymbol{\sigma}|-\left|\boldsymbol{\nu}_{-}\right|} \partial^{\boldsymbol{k}} f\right\|_{q, \mathbf{s}_{\phi}}\right\} .
\end{aligned}
$$

Moreover, if $q=\infty$ then 32 does not require $\mathrm{H}_{\text {国. }}$, 
Proof $\mathrm{By} \mathrm{H}_{\mathcal{M}}$, 15 and $\# \mathrm{~A}_{\omega} \leq C_{\mathrm{E}}$ we have

$$
\begin{aligned}
\left\|\partial^{\boldsymbol{\sigma}}(f-\aleph f)\right\|_{\infty, \omega} \leq C_{\lambda} C_{\Phi} C_{\mathrm{s}}^{|\boldsymbol{\sigma}|} C_{\Pi} C_{\mathrm{E}} C_{\mathcal{M}} & \\
& {\left[\sum_{\boldsymbol{k} \in K_{\boldsymbol{\sigma}}} \boldsymbol{h}_{\omega}^{\boldsymbol{k}-\boldsymbol{\sigma}+\boldsymbol{\nu}}\left\|\partial^{\boldsymbol{k}} f\right\|_{q, \omega}+\max _{\phi \in \mathrm{A}_{\omega}} \sum_{\boldsymbol{k} \in K_{\mathbf{0}}} \boldsymbol{h}_{\mathbf{s}_{\phi}}^{\boldsymbol{k}-\boldsymbol{\sigma}+\boldsymbol{\nu}}\left\|\partial^{\boldsymbol{k}} f\right\|_{q, \mathbf{s}_{\phi}}\right] . }
\end{aligned}
$$

If $q=\infty$ and $\boldsymbol{\sigma}=\mathbf{0}$, then $\boldsymbol{\nu}=\mathbf{0}$ and $\boldsymbol{h}_{\omega}^{\boldsymbol{k}} \leq \boldsymbol{h}_{\mathbf{s}_{\phi}}^{\boldsymbol{k}}$, so that the first term in brackets is bounded by the second and we get (31).

If $q<\infty$ or $\boldsymbol{\sigma} \neq \mathbf{0}$ we use $(4), \mathrm{H}_{[\mathbf{w}}$ and $\mathrm{H}_{[\mathbf{s}}$ to obtain

$$
\begin{aligned}
& \boldsymbol{h}_{\omega}^{\boldsymbol{k}-\boldsymbol{\sigma}+\boldsymbol{\nu}} \leq C_{\varpi}^{|\boldsymbol{\nu}|}\left(\max \boldsymbol{h}_{\mathbf{s}_{\phi}}\right)^{|\boldsymbol{k}|-|\boldsymbol{\sigma}|-|\boldsymbol{\nu}|}, \quad \boldsymbol{k} \geq \boldsymbol{\sigma}, \\
& \boldsymbol{h}_{\mathbf{s}_{\phi}}^{\boldsymbol{k}-\boldsymbol{\sigma}+\boldsymbol{\nu}} \leq C_{[\mathbf{S}]}^{|\boldsymbol{\sigma}|+|\boldsymbol{\nu}|}\left(\max \boldsymbol{h}_{\mathbf{s}_{\phi}}\right)^{|\boldsymbol{k}|-|\boldsymbol{\sigma}|-|\boldsymbol{\nu}|}
\end{aligned}
$$

and inserting these in 33 gives 32 . If $q=\infty$ then $\mathrm{H}_{\square}$ is not required.

\subsection{Mesh assumptions}

The assumption $\mathrm{H}_{\mathcal{M}}$ can be replaced by one of the following

$\mathrm{H}_{\mathcal{M}}^{\#} \# \mathrm{M}_{\phi} \leq C_{\#}$, for all $\phi \in \Phi, \#$ elements in $\operatorname{supp} \phi$;

$\mathrm{H}_{\mathcal{M}}^{\mathrm{s} \omega} \boldsymbol{h}_{\mathbf{s}_{\phi}} \leq C_{\mathrm{s} \omega} \boldsymbol{h}_{\omega}$, for all $\omega \in \mathcal{M}$ and $\phi \in \mathrm{A}_{\omega}$, local quasi-uniformity.

We show in Proposition 3 that $\mathrm{H}_{\mathcal{M}}^{\#}$ implies $\mathrm{H}_{\mathcal{M}}$ for $\gamma \geq-\mathbf{1} / p$ and that $\mathrm{H}_{\mathcal{M}}^{\mathrm{s} \omega}$ implies

\begin{tabular}{|c|c|c|}
\hline \multicolumn{2}{|c|}{ conditions } & $C_{\mathcal{M}}$ \\
\hline $\mathbf{0} \leq \gamma$ & & 1 \\
\hline$-1 / p \leq \gamma$ & $\mathrm{H}_{\mathcal{M}}^{\#}$ & $C_{\#}^{-\min \gamma}$ \\
\hline$\gamma \in \mathbb{R}^{n}$ & $\mathrm{H}_{\mathcal{M}}^{\mathrm{s \omega}}$ & $C_{\mathbf{s} \omega}^{|(\gamma+\mathbf{1} / p)-|+\frac{n}{p}}$ \\
\hline
\end{tabular}
$\mathrm{H}_{\mathcal{M}}$ for all values of $\gamma$.

Proposition 3 The conditions listed in the following table imply $\mathrm{H}_{\mathcal{M}}$ with the corresponding constants $C_{\mathcal{M}}$.

Proof First we note that for all $\boldsymbol{\alpha} \geq 1$ we have

$$
\sum_{\omega \in \mathbb{M}_{\phi}} \frac{\boldsymbol{h}_{\omega}^{\boldsymbol{\alpha}}}{\boldsymbol{h}_{\mathbf{s}_{\phi}}^{\boldsymbol{\alpha}}}=\sum_{\omega \in \mathrm{M}_{\phi}} \prod_{i=1}^{n}\left(\frac{h_{\omega, i}}{h_{\mathbf{s}_{\phi}, i}}\right)^{\alpha_{i}} \leq \sum_{\omega \subseteq \mathbf{s}_{\phi}} \frac{\boldsymbol{h}_{\omega}^{\mathbf{1}}}{\boldsymbol{h}_{\mathbf{s}_{\phi}}^{\mathbf{1}}}=\frac{\mu(\operatorname{supp} \phi)}{\mu\left(\mathrm{b}\left(\mathbf{s}_{\phi}\right)\right)} \leq 1
$$

This gives the first case as $\boldsymbol{\alpha}=\gamma p+\mathbf{1} \geq \mathbf{1}$. For $\gamma \geq \mathbf{- 1 / p}$, we use 10 with $e=\min \gamma p+1, \mathrm{H}_{\mathcal{M}}^{\#}$ and with $\boldsymbol{\alpha}=(\gamma p+\mathbf{1}) /(\min \gamma p+1) \geq \mathbf{1}$ we obtain

$$
\sum_{\omega \in \mathrm{M}_{\phi}} \frac{\boldsymbol{h}_{\omega}^{\gamma p+1}}{\boldsymbol{h}_{\mathbf{s}_{\phi}}^{\gamma p+1}} \leq \# \mathrm{M}_{\phi}-\min \gamma p\left(\sum_{\omega \in \mathrm{M}_{\phi}} \frac{\boldsymbol{h}_{\omega}^{\boldsymbol{\alpha}}}{\boldsymbol{h}_{\mathbf{s}_{\phi}}^{\boldsymbol{\alpha}}}\right)^{\min \gamma p+1} \leq C_{\#}^{-\min \gamma p} .
$$

This shows the second case. For the last case, if $\mathrm{H}_{\mathcal{M}}^{\mathrm{s \omega}}$ holds then

$$
\mu\left(\mathbf{s}_{\phi}\right) \geq \sum_{\omega \in \mathbb{M}_{\phi}} \mu(\omega) \geq \# \mathrm{M}_{\phi} \min _{\omega \in \mathbb{M}_{\phi}} \mu(\omega) \geq \# \mathrm{M}_{\phi} C_{\mathrm{s} \omega}^{-n} \mu\left(\mathbf{s}_{\phi}\right) .
$$




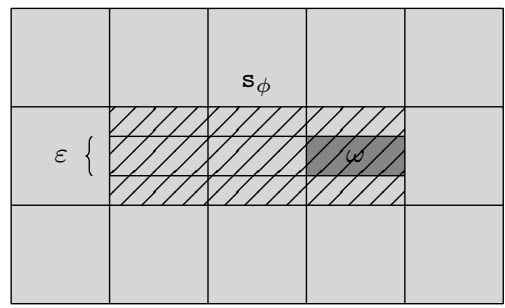

Fig. 5 A family of LR spaces on which $\mathrm{H}_{\mathcal{M}}^{\#}$ and $\mathrm{H}_{[\mathbf{S}}$ hold uniformly, but $C_{\varpi}$ is not bounded.

Therefore $\mathrm{H}_{\mathcal{M}}^{\#}$ holds with $C_{\#}=C_{\mathrm{s} \omega}^{n}$. Using $\mathrm{H}_{\mathcal{M}}^{\mathrm{s} \omega}$ again and with $\boldsymbol{\alpha}=\gamma p+\mathbf{1}$ we have

$$
\sum_{\omega \in \mathrm{M}_{\phi}} \frac{\boldsymbol{h}_{\omega}^{\boldsymbol{\alpha}}}{\boldsymbol{h}_{\mathbf{s}_{\phi}}^{\boldsymbol{\alpha}}} \leq \# \mathrm{M}_{\phi} \max _{\omega \in \mathrm{M}_{\phi}} \frac{\boldsymbol{h}_{\omega}^{\boldsymbol{\alpha}}}{\boldsymbol{h}_{\mathbf{s}_{\phi}}^{\boldsymbol{\alpha}}} \leq C_{\mathbf{s} \omega}^{n} C_{\mathbf{s} \omega}^{|\boldsymbol{\alpha}-|} .
$$

An interesting observation is that $\mathrm{H}_{\mathcal{M}}^{\#}$ and $\mathrm{H}_{\mathcal{M}}^{\mathrm{s \omega}}$ influence the relation between $\mathrm{H}_{[\omega}$ and $\mathrm{H}_{[\mathbf{s}}$ Assuming $\mathrm{H}_{\mathcal{M}}^{\#}$ we have that $\mathrm{H}_{[\mathbf{\omega}}$ implies $\mathrm{H}_{[\mathbf{s}}$ Assuming $\mathrm{H}_{\mathcal{M}}^{\mathrm{s \omega}}$ the two shape regularity assumptions are equivalent. This is proved in Proposition 4.

Proposition 4 We have

$$
\begin{aligned}
\mathrm{H}_{\mathcal{M}}^{\#} & \Rightarrow\left(\mathrm{H}_{[\omega]} \Rightarrow \mathrm{H}_{[\mathbf{s}}\right), \\
\mathrm{H}_{\mathcal{M}}^{\mathrm{s} \omega} & \Rightarrow\left(\mathrm{H}_{[} \Leftrightarrow \mathrm{H}_{[\mathbf{s}}\right) \text { and } C_{\mathrm{s} \omega}^{-1} C_{[\mathbf{s}} \leq C_{\underline{\omega}} \leq C_{\mathrm{s} \omega} C_{[\mathbf{s}} .
\end{aligned}
$$

Proof Let $\omega \subseteq \mathbf{s}_{\phi}$ be the element having the longest edge $h_{\omega, i}$. Then by $\mathrm{H}_{\mathcal{M}}^{\#}$ and $\mathrm{H}_{\omega}$

$$
\max \boldsymbol{h}_{\mathbf{s}_{\phi}} \leq C_{\#} \max \boldsymbol{h}_{\omega} \leq C_{\#} C_{\omega} \min \boldsymbol{h}_{\omega} \leq C_{\#} C_{\omega} \min \boldsymbol{h}_{\mathbf{s}_{\phi}} .
$$

Let $i, j \in\{1, \ldots, n\}$ be such that $\min \boldsymbol{h}_{\omega}=h_{\omega, i}$ and $\min \boldsymbol{h}_{\mathbf{s}_{\phi}}=h_{\mathbf{s}_{\phi}, j}$. Then using $\mathrm{H}_{\mathcal{M}}^{\mathrm{s} \omega}$ and $\mathrm{H}_{[\mathbf{s}}$ we have

$$
\begin{aligned}
& \max \boldsymbol{h}_{\omega} \leq \max \boldsymbol{h}_{\mathbf{s}_{\phi}} \leq C_{\mathbf{S}} \min \boldsymbol{h}_{\mathbf{s}_{\phi}} \\
& \quad=C_{[\mathbf{S}} h_{\mathbf{s}_{\phi}, j} \leq C_{[\mathbf{S}} h_{\mathbf{s}_{\phi}, i} \leq C_{[\mathbf{S}} C_{\mathbf{s} \omega} h_{\omega, i}=C_{[\mathbf{S}} C_{\mathbf{s} \omega} \min \boldsymbol{h}_{\omega}
\end{aligned}
$$

and $\mathrm{H}_{[}$follows. Similarly with the same $i, j$ and using $\left.\mathrm{H}_{\mathcal{M}}^{\mathrm{s \omega}}\right]$ and $\mathrm{H}_{[}$we have

$$
\begin{aligned}
& \max \boldsymbol{h}_{\mathbf{s}_{\phi}} \leq C_{\mathbf{s} \omega} \max \boldsymbol{h}_{\omega} \leq C_{\varpi} C_{\mathbf{s} \omega} \min \boldsymbol{h}_{\omega} \\
& \quad=C_{\varpi} C_{\mathbf{s} \omega} h_{\omega, i} \leq C_{\varpi} C_{\mathbf{s} \omega} h_{\omega, j} \leq C_{\varpi} C_{\mathbf{s} \omega} h_{\mathbf{s}_{\phi}, j}=C_{\varpi} C_{\mathbf{s} \omega} \min \boldsymbol{h}_{\mathbf{s}_{\phi}}
\end{aligned}
$$

and $\mathrm{H}_{[\mathbf{S}}$ follows.

Fig. 5 shows a family of LR spaces [8] 16 on which $\mathrm{H}_{\mathcal{M}}^{\#}$ and $\mathrm{H}_{[\mathrm{S}}$ hold uniformly, but $C_{\omega}$ is not bounded. The element $\omega(\square)$ has size $\boldsymbol{h}_{\omega}=(1, \varepsilon)$ and $\operatorname{supp} \phi=\mathbf{s}_{\phi}$ $(/ /)$ ) has size $\boldsymbol{h}_{\mathbf{s}_{\phi}}=(3,1)$. It can be shown that $\mathrm{H}_{\mathcal{M}}^{\#}$ holds with $C_{\#}=13, \mathrm{H}_{\varpi}$ holds with $C_{[\omega}=\varepsilon^{-1}$ and $\mathrm{H}_{[\mathbf{s}}$ holds with $C_{[\mathbf{s}}=3$. By letting $\varepsilon \rightarrow 0$ we observe that no bound for $C_{\omega}$ can be a function of $C_{\#}$ and $C_{[\mathbf{s}}$. 


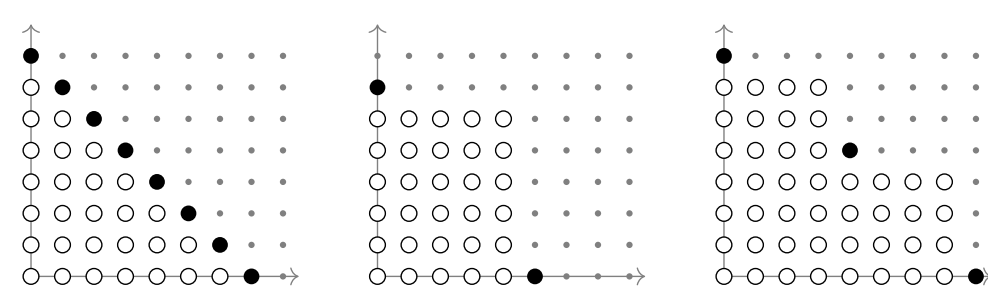

Fig. 6 Examples of index sets $A(0)$ and their bases $A_{B}(\bullet)$ in $\mathbb{N}^{2}$.

\section{Polynomial approximation}

This section describes collections of operators $\Pi_{\omega}$ that satisfy $\mathrm{H}_{\Pi}$. The construction is based on averaged Taylor expansion operators. To an index set $A \subseteq \mathbb{N}^{n}$ and a weight function $\psi \in L^{1}(\Omega)$ such that $\int \psi=1$ we associate the operator $T_{A, \psi}: \mathcal{C}^{\infty}(\Omega) \rightarrow \mathbb{P}_{A}$ defined by

$$
T_{A, \psi} f(\boldsymbol{x}):=\sum_{\boldsymbol{\alpha} \in A} \int_{\Omega} \psi(\boldsymbol{y}) \frac{(\boldsymbol{x}-\boldsymbol{y})^{\boldsymbol{\alpha}}}{\boldsymbol{\alpha} !} \partial^{\boldsymbol{\alpha}} f(\boldsymbol{y}) d \boldsymbol{y} .
$$

It is required that $\mathbb{P}_{A}$ is translation invariant:

$$
\forall \boldsymbol{y} \in \mathbb{R}^{n}, \quad \mathbb{P}_{A}:=\operatorname{span}\left\{\boldsymbol{x}^{\boldsymbol{\alpha}}: \boldsymbol{\alpha} \in A\right\}=\operatorname{span}\left\{(\boldsymbol{x}-\boldsymbol{y})^{\boldsymbol{\alpha}}: \boldsymbol{\alpha} \in A\right\} .
$$

Different choices are possible. The error bounds in terms of Sobolev and reduced seminorms use $A=\left\{\boldsymbol{\alpha} \in \mathbb{N}^{n}:|\boldsymbol{\alpha}| \leq d\right\}$ and $A=\left\{\boldsymbol{\alpha} \in \mathbb{N}^{n}: \boldsymbol{\alpha} \leq \boldsymbol{d}\right\}$, respectively.

The operators $T_{A, \psi}$ are defined on $\mathcal{C}^{\infty}(\Omega)$ and are uniquely extended to a Sobolev space $W$, provided $\mathcal{C}^{\infty}(\Omega)$ is dense in $W$ and $T_{A, \psi}$ is continuous with respect to the norm of $W$. We also use the symbol $T_{A, \psi}$ for such extensions.

In the following we recall and prove the elementary properties of averaged Taylor expansions. For the approximation properties we refer to [17. We will need the translations $A-\sigma$ of $A$ and the base $A_{B}$ of $A$ defined as follows

$$
\begin{aligned}
& A-\boldsymbol{\sigma}:=\left\{\boldsymbol{\alpha}-\boldsymbol{\sigma} \in \mathbb{N}^{n}: \boldsymbol{\alpha} \in A\right\}, \\
& A_{B}:=\left\{\boldsymbol{\beta} \in \mathbb{N}^{n} \backslash A: \boldsymbol{k} \in \mathbb{N}^{n} \backslash A \text { and } \boldsymbol{k} \leq \boldsymbol{\beta} \Rightarrow \boldsymbol{k}=\boldsymbol{\beta}\right\} .
\end{aligned}
$$

Fig. 6 contains some examples of $A$ and $A_{B}$.

\subsection{General properties}

Proposition $5 T_{A, \psi}$ is a projector, i.e., for all $g \in \mathbb{P}_{A}, T_{A, \psi} g=g$.

Proof The Taylor expansion at the point $\boldsymbol{y}$ of $g \in \mathbb{P}_{A}$ is a polynomial that has the same partial derivatives as $g$. Therefore

$$
g(\boldsymbol{x})=\sum_{\boldsymbol{\alpha} \in A} \frac{(\boldsymbol{x}-\boldsymbol{y})^{\boldsymbol{\alpha}}}{\boldsymbol{\alpha} !} \partial^{\boldsymbol{\alpha}} g(\boldsymbol{y})
$$

and since $\int \psi=1$ we have $T_{A, \psi} g(\boldsymbol{x})=g(\boldsymbol{x})$. 
Proposition $\mathbf{6}$ For all $\boldsymbol{\sigma} \geq \mathbf{0}$ and $f \in \mathcal{C}^{\infty}$ we have

$$
\partial^{\sigma} T_{A, \psi} f=T_{A-\sigma, \psi} \partial^{\sigma} f
$$

Proof The derivatives are with respect to $\boldsymbol{x}$ and can be computed inside the integral. We obtain

$$
\begin{aligned}
\partial^{\boldsymbol{\sigma}} T_{A, \psi} f(\boldsymbol{x}) & =\sum_{\substack{\boldsymbol{\alpha} \in A \\
\boldsymbol{\alpha} \geq \boldsymbol{\sigma}}} \int_{\Omega} \psi(\boldsymbol{y}) \frac{(\boldsymbol{x}-\boldsymbol{y})^{\boldsymbol{\alpha}-\boldsymbol{\sigma}}}{(\boldsymbol{\alpha}-\boldsymbol{\sigma}) !} \partial^{\boldsymbol{\alpha}} f(\boldsymbol{y}) d \boldsymbol{y} \\
& =\sum_{\boldsymbol{\beta} \in A-\boldsymbol{\sigma}} \int_{\Omega} \psi(\boldsymbol{y}) \frac{(\boldsymbol{x}-\boldsymbol{y})^{\boldsymbol{\beta}}}{\boldsymbol{\beta} !} \partial^{\boldsymbol{\beta}} \partial^{\boldsymbol{\sigma}} f(\boldsymbol{y}) d \boldsymbol{y}=T_{A-\boldsymbol{\sigma}, \psi} \partial^{\boldsymbol{\sigma}} f(\boldsymbol{x}) .
\end{aligned}
$$

Lemma 1 If $\psi \in \mathcal{C}_{0}^{\max }{ }^{A}(\omega)$ then

$$
T_{A, \psi} f(\boldsymbol{x})=\sum_{\boldsymbol{\alpha} \in A} C_{\boldsymbol{\alpha}, A} \int_{\omega} \partial^{\boldsymbol{\alpha}} \psi(\boldsymbol{y}) \frac{(\boldsymbol{x}-\boldsymbol{y})^{\boldsymbol{\alpha}}}{\boldsymbol{\alpha} !} f(\boldsymbol{y}) d \boldsymbol{y} .
$$

where $C_{\boldsymbol{\alpha}, A}:=(-1)^{|\boldsymbol{\alpha}|} \sum_{\substack{\boldsymbol{\beta} \in A \\ \boldsymbol{\alpha} \geq \boldsymbol{\alpha}}}\left(\begin{array}{c}\boldsymbol{\beta} \\ \boldsymbol{\alpha}\end{array}\right)$.

Proof Writing (34) with $\boldsymbol{\beta}$ in place of $\boldsymbol{\alpha}$, integrating each term by parts, noting that the boundary terms vanish and expanding $\partial^{\boldsymbol{\beta}}\left(\psi(\boldsymbol{y})(\boldsymbol{x}-\boldsymbol{y})^{\boldsymbol{\beta}}\right)$ leads to

$$
\begin{aligned}
T_{A, \psi} f(\boldsymbol{x}) & =\sum_{\boldsymbol{\beta} \in A}(-1)^{|\boldsymbol{\beta}|} \sum_{\substack{\boldsymbol{\alpha} \in A \\
\boldsymbol{\alpha} \leq \boldsymbol{\beta}}}\left(\begin{array}{l}
\boldsymbol{\beta} \\
\boldsymbol{\alpha}
\end{array}\right) \int_{\omega} \partial^{\boldsymbol{\alpha}} \psi(\boldsymbol{y}) \frac{(-1)^{|\boldsymbol{\beta}|-|\boldsymbol{\alpha}|}(\boldsymbol{x}-\boldsymbol{y})^{\boldsymbol{\alpha}}}{\boldsymbol{\alpha} !} f(\boldsymbol{y}) d \boldsymbol{y} \\
& =\sum_{\boldsymbol{\alpha} \in A}\left[(-1)^{|\boldsymbol{\alpha}|} \sum_{\substack{\boldsymbol{\beta} \in A \\
\boldsymbol{\beta} \geq \boldsymbol{\alpha}}}\left(\begin{array}{l}
\boldsymbol{\beta} \\
\boldsymbol{\alpha}
\end{array}\right)\right] \int_{\omega} \partial^{\boldsymbol{\alpha}} \psi(\boldsymbol{y}) \frac{(\boldsymbol{x}-\boldsymbol{y})^{\boldsymbol{\alpha}}}{\boldsymbol{\alpha} !} f(\boldsymbol{y}) d \boldsymbol{y} .
\end{aligned}
$$

Lemma 2 For all weights $\psi$ with $\operatorname{supp} \psi \subseteq \omega$, and box $\eta \subset \mathbb{R}^{n}$ the operator $T_{A, \psi}$ is continuous, meaning that for all $v$ such that $\partial^{\boldsymbol{\alpha}} v \in L^{q}(\omega), \boldsymbol{\alpha} \in A$ we have

$$
\left\|T_{A, \psi} v\right\|_{p, \eta} \leq\|\psi\|_{q^{\prime}} \sum_{\boldsymbol{\alpha} \in A} \frac{\boldsymbol{h}_{\eta}^{\boldsymbol{\alpha}+\mathbf{1} / p}}{\boldsymbol{\alpha} !}\left\|\partial^{\boldsymbol{\alpha}} v\right\|_{q, \omega} .
$$

Moreover, if $\psi \in \mathcal{C}_{0}^{\max A}(\omega)$ then for any $v \in L^{q}(\omega)$

$$
\left\|T_{A, \psi} v\right\|_{p, \eta} \leq\left(\sum_{\boldsymbol{\alpha} \in A}\left|C_{\boldsymbol{\alpha}, A}\right| \frac{\boldsymbol{h}_{\eta}^{\boldsymbol{\alpha}+\mathbf{1} / p}}{\boldsymbol{\alpha} !}\left\|\partial^{\boldsymbol{\alpha}} \psi\right\|_{q^{\prime}, \omega}\right)\|v\|_{q, \omega},
$$

where $C_{\boldsymbol{\alpha}, A}$ is from Lemma 1 
Proof First note that for $\boldsymbol{y} \in \eta$

$$
\left\|\frac{(\cdot-\boldsymbol{y})^{\boldsymbol{\alpha}}}{\boldsymbol{\alpha} !}\right\|_{p, \eta} \leq \frac{\boldsymbol{h}_{\eta}^{\boldsymbol{\alpha}+\mathbf{1} / p}}{\boldsymbol{\alpha} !} .
$$

Let $f_{\boldsymbol{\alpha}}(\boldsymbol{x}, \boldsymbol{y}):=\left|\psi(\boldsymbol{y})(\boldsymbol{x}-\boldsymbol{y})^{\boldsymbol{\alpha}}(\boldsymbol{\alpha} !)^{-1} \partial^{\boldsymbol{\alpha}} v(\boldsymbol{y})\right|$. Using Minkowski's integral inequality, see [2, Theorem 4, p. 21], the above and Hölder's inequality we have

$$
\begin{aligned}
\left\|T_{A, \psi} v\right\|_{p, \eta} & \leq\left\|\sum_{\boldsymbol{\alpha} \in A} \int_{\omega} f_{\boldsymbol{\alpha}}(\cdot, \boldsymbol{y}) d \boldsymbol{y}\right\|_{p, \eta} \leq \sum_{\boldsymbol{\alpha} \in A}\left\|\int_{\omega} f_{\boldsymbol{\alpha}}(\cdot, \boldsymbol{y}) d \boldsymbol{y}\right\|_{p, \eta} \\
& \leq \sum_{\boldsymbol{\alpha} \in A} \int_{\omega}\left\|f_{\boldsymbol{\alpha}}(\cdot, \boldsymbol{y})\right\|_{p, \eta} d \boldsymbol{y} \leq \sum_{\boldsymbol{\alpha} \in A} \frac{\boldsymbol{h}_{\eta}^{\boldsymbol{\alpha}+\mathbf{1} / p}}{\boldsymbol{\alpha} !}\|\psi\|_{q^{\prime}, \omega}\left\|\partial^{\boldsymbol{\alpha}} v\right\|_{q, \omega} .
\end{aligned}
$$

Similarly, with $f_{\boldsymbol{\alpha}}(\boldsymbol{x}, \boldsymbol{y})=\left|C_{\boldsymbol{\alpha}, A} \partial^{\boldsymbol{\alpha}} \psi(\boldsymbol{y})(\boldsymbol{x}-\boldsymbol{y})^{\boldsymbol{\alpha}}(\boldsymbol{\alpha} !)^{-1} v(\boldsymbol{y})\right|$ we obtain 41).

\subsection{Approximation and $\mathrm{H}_{\Pi}$}

In this subsection we prove that the operators $T_{A, \psi_{\omega}}$ satisfy $\mathrm{H}_{\Pi}$. Here for any box $\eta$, the function $\psi_{\eta}$ is

$$
\psi_{\eta}:=\mu(\eta)^{-1}\left(\hat{\psi} \circ M_{\eta}\right),
$$

where $M_{\eta}$ is the orientation preserving affine bijection $\eta \rightarrow[\mathbf{0}, \mathbf{1}]$ and $\hat{\psi}:[\mathbf{0}, \mathbf{1}] \rightarrow \mathbb{R}$ is a fixed function such that $\int \hat{\psi}=1$. The result requires the following assumption on the $\mathbf{s}_{\phi}$

$\mathrm{H}_{\star}$ each $\mathbf{s}_{\phi}, \phi \in \Phi$, is star shaped with respect to a box $\zeta_{\phi}$ and $\boldsymbol{h}_{\mathbf{s}_{\phi}} \leq C_{\star} \boldsymbol{h}_{\zeta_{\phi}}$.

If the $\mathbf{s}_{\phi}$ are boxes then taking $\zeta_{\phi}=\mathbf{s}_{\phi}$ implies $\mathrm{H}_{\star}$ with $C_{\star}=1$.

The main idea is to specialize the approximation results from Dupont and Scott [17] to truncated boxes satisfying $\mathrm{H}_{\star}$. The error bounds in [17] for a domain $\eta$ star shaped with respect to $\operatorname{supp} \psi$ have the general form

$$
\left\|f-T_{A, \psi} f\right\|_{p, \eta} \leq C \sum_{\boldsymbol{\alpha} \in A_{B}}\left\|\partial^{\alpha} f\right\|_{q, \eta} .
$$

The assumptions in [17] are equivalent to $(1 / q, 1 / p) \in R_{|\boldsymbol{\alpha}|}, \boldsymbol{\alpha} \in A_{B}$, where

$$
R_{r}:= \begin{cases}\left\{\left(\frac{1}{q}, \frac{1}{p}\right): \frac{1}{p}-\frac{1}{q}+\frac{r}{n} \geq 0\right\} \backslash\left\{\left(\frac{r}{n}, 0\right),\left(1,1-\frac{r}{n}\right)\right\} & r<n \\ {[\mathbf{0}, \mathbf{1}]} & r \geq n .\end{cases}
$$

See Fig. 7. Note that $R_{r}$ almost coincide with the domain of validity of the Sobolev embedding $W^{r, q}(\stackrel{\Omega}{\Omega}) \rightarrow L^{p}(\Omega)$. In fact the embedding also holds at the point $(1,1-r / n)$ [1, Theorem 4.12 and Remark 4.13 point 3].

Proposition 7 Let $\hat{\psi} \in \mathcal{C}_{0}^{\max A}([\mathbf{0}, \mathbf{1}])$ and $\int \hat{\psi}=1$. Suppose $A \subseteq \mathbb{N}^{n}$ satisfies (35), and that for all $\boldsymbol{\alpha} \in(A-\boldsymbol{\sigma})_{B}$ we have $(1 / q, 1 / p) \in R_{|\boldsymbol{\alpha}|}$. Then there exists constants $C_{\boldsymbol{\alpha}}$, such that for all truncated boxes $\eta$ star shaped with respect to a box $\zeta$ as in $\mathrm{H}_{\star}$, and for all $f$ with $\partial^{\boldsymbol{\alpha}+\boldsymbol{\sigma}} f \in L^{q}(\eta)$ we have

$$
\left\|\partial^{\boldsymbol{\sigma}}\left(f-T_{A, \psi_{\zeta}} f\right)\right\|_{p, \eta} \leq \sum_{\boldsymbol{\alpha} \in(A-\boldsymbol{\sigma})_{B}} C_{\boldsymbol{\alpha}}\left\|\boldsymbol{h}_{\eta}^{\boldsymbol{\alpha}+\boldsymbol{\nu}} \partial^{\boldsymbol{\alpha}+\boldsymbol{\sigma}} f\right\|_{q, \eta} .
$$



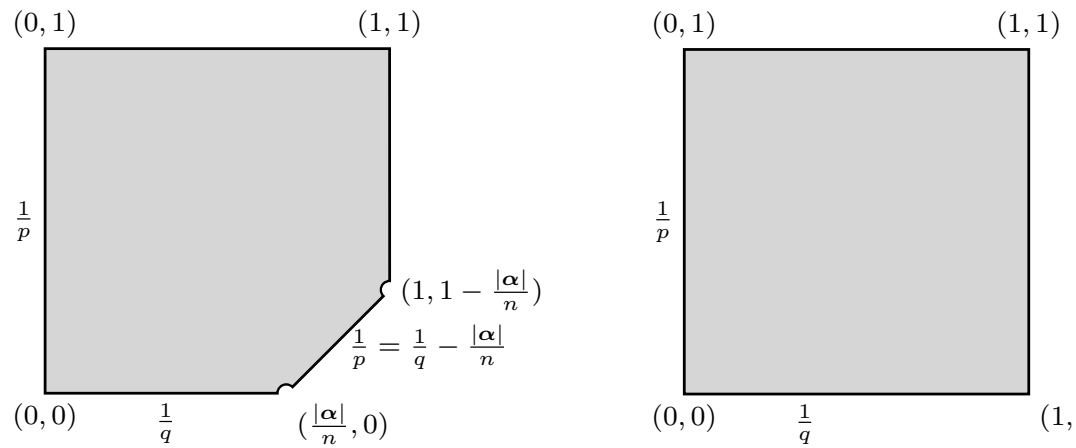

Fig. 7 On the left, the region $R_{|\boldsymbol{\alpha}|}$ for $|\boldsymbol{\alpha}|<n$. On the right the case $|\boldsymbol{\alpha}| \geq n$.

Proof First we consider $\boldsymbol{\sigma}=\mathbf{0}$ and a truncated box $\eta=\overline{[\mathbf{0 , 1}] \backslash \beta}$ where $\beta$ is a box. The diameter of $\eta$ is $\sqrt{n}$ because it contains two opposite vertices of $[\mathbf{0 , 1}]$.

For a given $\zeta$ this is a special case of [17, Theorem 4.2] and corresponds to the following substitutions and equivalency

\begin{tabular}{r|rrrrrrrrr} 
Dupont-Scott & $m$ & $\boldsymbol{\beta}$ & $q$ & $p_{\boldsymbol{\alpha}}$ & $A^{0}$ & $A_{-}$ & $D$ & $d$ & $\mu_{\boldsymbol{\alpha}}>0$ \\
here & 0 & $\mathbf{0}$ & $p$ & $q$ & $A$ & $A_{B}$ & $\eta$ & $\sqrt{n}$ & $\left(\frac{1}{q}, \frac{1}{p}\right) \in R_{|\boldsymbol{\alpha}|}$
\end{tabular}

Note that in [17] the floor of $r \in \mathbb{R}$ is written as $\lceil r\rceil$.

The $C_{\boldsymbol{\alpha}}$ provided by [17] depend on $\psi_{\zeta}, p, q$. To find $C_{\boldsymbol{\alpha}}$ independent of $\zeta$ we use a compactness argument. By Proposition 2, and since $\psi_{\zeta} \in \mathcal{C}_{0}^{\max } A(\zeta)$, $T_{A, \psi_{\zeta}}$ depends continuously on the size and position of $\zeta$. These are described respectively by $\boldsymbol{h}_{\zeta} \in\left[C_{\star}^{-1} \mathbf{1}, \mathbf{1}\right] \subseteq \mathbb{R}^{n}$ and a vector in $\left[\mathbf{0}, \mathbf{1}-\boldsymbol{h}_{\zeta}\right] \subseteq \mathbb{R}^{n}$. Consequently the constants are bounded on a compact set and they have a maximum.

For other truncated boxes $\eta$ we apply a scaling and a translation. For $\boldsymbol{\sigma} \neq \mathbf{0}$ we use Proposition 6 in the form $\partial^{\sigma}\left(f-T_{A, \psi} f\right)=g-T_{A-\sigma, \psi} g, g=\partial^{\sigma} f$.

We next consider a constant weight. Proposition 7 does not apply since $\psi \notin$ $\mathcal{C}_{0}^{\max A}$, nevertheless we obtain an error bound with an explicit constant.

Proposition 8 Let $\eta$ be a box. For $1 \leq p, q \leq \infty, d \geq n, A=\left\{\boldsymbol{\alpha} \in \mathbb{N}^{n}:|\boldsymbol{\alpha}| \leq d\right\}$ and $\psi_{\eta}(\boldsymbol{x}):=\mu(\eta)^{-1} \mathbb{1}_{\eta}(\boldsymbol{x})$ we have

$$
\left\|\partial^{\boldsymbol{\sigma}}\left(f-T_{A, \psi_{\eta}} f\right)\right\|_{p, \eta} \leq \sum_{|\boldsymbol{\alpha}|=d+1-|\boldsymbol{\sigma}|} \frac{(d+1-|\boldsymbol{\sigma}|) n^{\frac{d-1}{2}}}{\boldsymbol{\alpha} !}\left\|\boldsymbol{h}_{\eta}^{\boldsymbol{\alpha}+\boldsymbol{\nu}} \partial^{\boldsymbol{\alpha}+\boldsymbol{\sigma}} f\right\|_{q, \eta} .
$$

Proof Suppose $\boldsymbol{\sigma}=\mathbf{0}$. It is enough to prove the case $p=\infty$ and $q=1$ because $\left\|f-T_{A, \psi_{\eta}} f\right\|_{p,[\mathbf{0}, \mathbf{1}]} \leq\left\|f-T_{A, \psi_{\eta}} f\right\|_{\infty,[\mathbf{0 , 1}]}$ and $\left\|\partial^{\boldsymbol{\alpha}} f\right\|_{1,[\mathbf{0}, \mathbf{1}]} \leq\left\|\partial^{\boldsymbol{\alpha}} f\right\|_{q,[\mathbf{0}, \mathbf{1}]}$. Using Sobolev representation [17, Section 3], that holds also for $\psi \notin \mathcal{C}_{0}^{\max } A$, we have

$$
\left(f-T_{A, \psi_{\eta}} f\right)(\boldsymbol{x})=\sum_{|\boldsymbol{\alpha}|=d+1} \frac{d+1}{\boldsymbol{\alpha} !} \int_{[\mathbf{0}, \mathbf{1}]} K_{\boldsymbol{\alpha}}(\boldsymbol{x}, \boldsymbol{y}) \partial^{\boldsymbol{\alpha}} f(\boldsymbol{y}) d \boldsymbol{y}
$$

where $K_{\boldsymbol{\alpha}}(\boldsymbol{x}, \boldsymbol{y}):=(\boldsymbol{x}-\boldsymbol{y})^{\boldsymbol{\alpha}} \int_{[0,1]} s^{-n-1} \psi_{\eta}\left(\boldsymbol{x}+s^{-1}(\boldsymbol{y}-\boldsymbol{x})\right) d s$. The integrand in $K_{\boldsymbol{\alpha}}$ is 0 for $\boldsymbol{x}+s^{-1}(\boldsymbol{y}-\boldsymbol{x}) \notin \operatorname{supp} \psi_{\eta}$, in particular for $s \leq n^{-1 / 2}\|\boldsymbol{x}-\boldsymbol{y}\|$. For 
$\boldsymbol{x}, \boldsymbol{y} \in[\mathbf{0}, \mathbf{1}]$ we have $\|\boldsymbol{x}-\boldsymbol{y}\| \leq n^{1 / 2}$ and we get

$$
\left|K_{\boldsymbol{\alpha}}(\boldsymbol{x}, \boldsymbol{y})\right| \leq\|\boldsymbol{x}-\boldsymbol{y}\|^{d+1} \int_{n^{-1 / 2}\|\boldsymbol{x}-\boldsymbol{y}\|}^{1} s^{-n-1} d s \leq n^{n / 2-1}\|\boldsymbol{x}-\boldsymbol{y}\|^{d+1-n} \leq n^{(d-1) / 2} .
$$

Inserting this estimate in 46 gives the result for $p=\infty$ and $q=1$. The case $\boldsymbol{\sigma} \neq \mathbf{0}$ follows as in Proposition 7 .

Theorem 4 Let $\hat{\psi} \in \mathcal{C}_{0}^{\max A}([\mathbf{0}, \mathbf{1}])$ and $\int \hat{\psi}=1$. Suppose $A \subseteq \mathbb{N}^{n}$ satisfies (35), and that for all $\boldsymbol{\alpha} \in(A-\boldsymbol{\sigma})_{B}$ we have $(1 / q, 1 / p) \in R_{|\boldsymbol{\alpha}|}$. Assume $\mathrm{H}_{\star}$. Then there exists a constant $C_{\Pi}$ such that the collection of operators $T_{A, \psi_{\omega}}$ satisfies $\mathrm{H}_{\Pi}$ with the following pairs $\left(K_{\boldsymbol{\beta}}, \gamma\right)$

$$
\begin{aligned}
& K_{\boldsymbol{\beta}}=\bigcup_{\boldsymbol{b} \in(A-\boldsymbol{\beta})}\left((A-\boldsymbol{b}-\boldsymbol{\beta})_{B}+\boldsymbol{b}\right) \quad \text { and } \quad \boldsymbol{\gamma}=-\mathbf{1} / q, \\
& K_{\boldsymbol{\beta}}=(A-\boldsymbol{\beta})_{B} \quad \text { and } \quad \gamma=-\max A-\mathbf{1} / q .
\end{aligned}
$$

In particular for $A=\left\{\boldsymbol{\alpha} \in \mathbb{N}^{n}:|\boldsymbol{\alpha}| \leq d\right\}$, 47) becomes

$$
K_{\boldsymbol{\beta}}=\{\boldsymbol{k} \geq \boldsymbol{\beta}:|\boldsymbol{k}|=d+1\}, \quad \gamma=-\mathbf{1} / q,
$$

and if in addition the $\mathbf{s}_{\phi}$ are boxes, i.e. $C_{\star}=1, \hat{\psi}=\mathbb{1}_{[\mathbf{0}, \mathbf{1}]}$ and $d-|\boldsymbol{\sigma}| \geq n$ then

$$
C_{\Pi}=2 \frac{\left(2 n^{\frac{3}{2}}\right)^{d+1}}{(d-|\boldsymbol{\sigma}|) !} .
$$

Proof We first consider the case $\boldsymbol{\beta}=\mathbf{0}$. For each $\omega \in \mathcal{M}$, we need to consider the approximation for both $\eta=\omega$ and $\eta=\mathbf{s}_{\phi}, \phi \in \mathrm{A}_{\omega}$. If $\eta=\omega$ then $\left[\mathrm{H}_{\Pi}\right.$ follows from equation 44) in Proposition 7

Suppose $\eta=\mathbf{s}_{\phi}$. By Proposition 5, $T_{A, \psi_{\omega}}$ is a projector on $\mathbb{P}_{A}$ and for all $g$ we have

$$
f-T_{A, \psi_{\omega}} f=(f-g)+T_{A, \psi_{\omega}}(f-g) .
$$

By 40 and $\left\|\psi_{\omega}\right\|_{q^{\prime}}=\boldsymbol{h}_{\omega}^{-1 / q}\|\hat{\psi}\|_{q^{\prime}}$ we deduce

$$
\left\|f-T_{A, \psi_{\omega}} f\right\|_{p, \eta} \leq\|f-g\|_{p, \eta}+\|\hat{\psi}\|_{q^{\prime}} \frac{\boldsymbol{h}_{\eta}^{\mathbf{1} / q}}{\boldsymbol{h}_{\omega}^{\mathbf{1} / q}} \sum_{\boldsymbol{b} \in A} \frac{\boldsymbol{h}_{\eta}^{\boldsymbol{b}+\boldsymbol{\nu}}}{\boldsymbol{b} !}\left\|\partial^{\boldsymbol{b}}(f-g)\right\|_{q, \eta} .
$$

We fix $g:=T_{A, \psi_{\zeta}} f \in \mathbb{P}_{A}$ where $\zeta \subseteq \eta$ is a box satisfying $\mathrm{H}_{\star}$ use Proposition 7 . and obtain

$$
\begin{aligned}
\left\|f-T_{A, \psi_{\omega}} f\right\|_{p, \eta} \leq & \sum_{\boldsymbol{\alpha} \in A_{B}} C_{\boldsymbol{\alpha}, p} \boldsymbol{h}_{\eta}^{\boldsymbol{\alpha}+\boldsymbol{\nu}}\left\|\partial^{\boldsymbol{\alpha}} f\right\|_{q, \eta} \\
& +\|\hat{\psi}\|_{q^{\prime}} \frac{\boldsymbol{h}_{\omega}^{-\mathbf{1} / q}}{\boldsymbol{h}_{\eta}^{-1 / q}} \sum_{\boldsymbol{b} \in A} \sum_{\boldsymbol{\alpha} \in(A-\boldsymbol{b})_{B}} C_{\boldsymbol{\alpha}, q} \frac{\boldsymbol{h}_{\eta}^{\boldsymbol{b}+\boldsymbol{\alpha}+\boldsymbol{\nu}}}{\boldsymbol{b} !}\left\|\partial^{\boldsymbol{\alpha}+\boldsymbol{b}} f\right\|_{q, \eta} \\
\leq & C_{\Pi} \frac{\boldsymbol{h}_{\omega}^{-\mathbf{1} / q}}{\boldsymbol{h}_{\eta}^{-\mathbf{1} / q}} \sum_{\boldsymbol{k} \in K_{\mathbf{0}}} \boldsymbol{h}_{\eta}^{\boldsymbol{k}+\boldsymbol{\nu}}\left\|\partial^{\boldsymbol{k}} f\right\|_{q, \eta}
\end{aligned}
$$


where we used $\boldsymbol{h}_{\omega}^{-\mathbf{1} / q} \boldsymbol{h}_{\eta}^{\mathbf{1} / q} \geq 1, \boldsymbol{\beta}=\mathbf{0}, K_{\mathbf{0}}$ as in (47) and $C_{\Pi}$ equals the maximum of the $C_{\boldsymbol{\alpha}, p}, \boldsymbol{\alpha} \in A_{B}$ and $\|\hat{\psi}\|_{q^{\prime}} C_{\boldsymbol{\alpha}, q}(\boldsymbol{b} !)^{-1}, \boldsymbol{b} \in A, \boldsymbol{\alpha} \in(A-\boldsymbol{b})_{B}$ times the maximum number of repetitions of a derivative of $f$.

To prove 49 we use Proposition 8 instead of Proposition 7 in 51 and obtain

$$
\left\|f-T_{A, \psi_{\omega}} f\right\|_{p, \eta} \leq E_{1}+E_{2}
$$

where

$$
\begin{aligned}
& E_{1}:=\sum_{|\boldsymbol{\alpha}|=d+1} \frac{(d+1) n^{\frac{d-1}{2}}}{\boldsymbol{\alpha} !} \boldsymbol{h}_{\eta}^{\boldsymbol{\alpha}+\boldsymbol{\nu}}\left\|\partial^{\boldsymbol{\alpha}} f\right\|_{q, \eta}, \\
& E_{2}:=\frac{\boldsymbol{h}_{\eta}^{\mathbf{1} / q}}{\boldsymbol{h}_{\omega}^{\mathbf{1} / q}} \sum_{|\boldsymbol{b}| \leq d} \frac{\boldsymbol{h}_{\eta}^{\boldsymbol{b}+\boldsymbol{\nu}}}{\boldsymbol{b} !} \sum_{|\boldsymbol{\alpha}|=d+1-|\boldsymbol{b}|} \frac{(d+1-|\boldsymbol{b}|) n^{\frac{d-1}{2}}}{\boldsymbol{\alpha} !} \boldsymbol{h}_{\eta}^{\boldsymbol{\alpha}}\left\|\partial^{\boldsymbol{\alpha}+\boldsymbol{b}} f\right\|_{q, \eta} .
\end{aligned}
$$

Setting $\boldsymbol{c}=\boldsymbol{b}+\boldsymbol{\alpha}$ and re-indexing the double sum leads to the upper bound

$$
E_{2} \leq \frac{\boldsymbol{h}_{\eta}^{\mathbf{1} / q}}{\boldsymbol{h}_{\omega}^{\mathbf{1} / q}} \sum_{|\boldsymbol{c}|=d+1}\left(\sum_{\boldsymbol{b} \leq \boldsymbol{c}} \frac{(d+1-|\boldsymbol{b}|) n^{\frac{d-1}{2}}}{(\boldsymbol{c}-\boldsymbol{b}) ! \boldsymbol{b} !}\right) \boldsymbol{h}_{\eta}^{\boldsymbol{c}+\boldsymbol{\nu}}\left\|\partial^{\boldsymbol{c}} f\right\|_{q, \eta} .
$$

Using $\sum_{\boldsymbol{b} \leq \boldsymbol{c}} \frac{\boldsymbol{c} !}{(\boldsymbol{c}-\boldsymbol{b}) ! \boldsymbol{b} !}=2^{|\boldsymbol{c}|}=2^{d+1}$ we get

$$
E_{2} \leq E_{3}:=\frac{\boldsymbol{h}_{\eta}^{\mathbf{1} / q}}{\boldsymbol{h}_{\omega}^{\mathbf{1} / q}} \sum_{|\boldsymbol{c}|=d+1} \frac{(d+1) 2^{d+1} n^{\frac{d-1}{2}}}{\boldsymbol{c} !} \boldsymbol{h}_{\eta}^{\boldsymbol{c}+\boldsymbol{\nu}}\left\|\partial^{\boldsymbol{c}} f\right\|_{q, \eta} .
$$

Finally, since $E_{1} \leq E_{3}$ and $(\boldsymbol{c} !)^{-1} \leq n^{d+1}(d+1) !^{-1}$ we obtain 49 for $\boldsymbol{\sigma}=\mathbf{0}$.

To obtain (48) we start from 41, set $g=T_{A, \psi_{\zeta}} f \in \mathbb{P}_{A}$ where $\zeta \subseteq \eta$ is a box satisfying $\mathrm{H}_{\star}$, use $\left\|\partial^{\boldsymbol{\alpha}} \psi_{\omega}\right\|_{q^{\prime}}=\boldsymbol{h}_{\omega}^{-1 / q} \boldsymbol{h}_{\omega}^{-\boldsymbol{\alpha}}\left\|\partial^{\boldsymbol{\alpha}} \hat{\psi}\right\|_{q^{\prime}}$, Proposition 7 and a scaling argument and obtain

$$
\begin{aligned}
\left\|f-T_{A, \psi_{\omega}} f\right\|_{p, \eta} & \leq\left(\sum_{\boldsymbol{\alpha} \in A} \frac{\left|C_{\boldsymbol{\alpha}, A}\right|}{\boldsymbol{\alpha} !} \frac{\boldsymbol{h}_{\omega}^{-\boldsymbol{\alpha}-\mathbf{1} / q}}{\boldsymbol{h}_{\eta}^{-\boldsymbol{\alpha}-\mathbf{1} / q}} \boldsymbol{h}_{\eta}^{\boldsymbol{\nu}}\left\|\partial^{\boldsymbol{\alpha}} \hat{\psi}\right\|_{q^{\prime}}\right)\|f-g\|_{q, \eta} \\
& \leq C_{\Pi} \frac{\boldsymbol{h}_{\omega}^{-\max A-\mathbf{1} / q}}{\boldsymbol{h}_{\eta}^{-\max A-\mathbf{1} / q}} \sum_{\boldsymbol{\alpha} \in A_{B}} \boldsymbol{h}_{\eta}^{\boldsymbol{\alpha}+\boldsymbol{\nu}}\left\|\partial^{\boldsymbol{\alpha}} f\right\|_{q, \eta}
\end{aligned}
$$

where $C_{\Pi}=\left(\sum_{\boldsymbol{\alpha} \in A}\left|C_{\boldsymbol{\alpha}, A}\right| \boldsymbol{\alpha} !^{-1}\left\|\partial^{\boldsymbol{\alpha}} \hat{\psi}\right\|_{q^{\prime}}\right) \max _{\boldsymbol{\alpha} \in A_{B}} C_{\boldsymbol{\alpha}}$.

The case $\boldsymbol{\beta}=\boldsymbol{\sigma} \neq \mathbf{0}$ follows from Proposition 6

\section{Sobolev and reduced seminorms}

We define two families of operators $\Pi_{\omega}^{S}$ and $\Pi_{\omega}^{R}$ of the form $T_{A, \psi_{\omega}}$ from which error bounds are derived in term of Sobolev and reduced seminorms, respectively. For each case we summarize the assumptions required by Theorems 1,2 and 3.

The family $\Pi_{\omega}^{S}$ is defined by $A=\left\{\boldsymbol{\alpha} \in \mathbb{N}^{n}:|\boldsymbol{\alpha}| \leq d\right\}, \psi_{\omega}=\mu(\omega)^{-1} \mathbb{1}_{\omega}$ and satisfy $\mathrm{H}_{\Pi}$ according to 47 with

$$
\boldsymbol{\gamma}=-\mathbf{1} / q, \quad K_{\mathbf{0}}=\{\boldsymbol{k}:|\boldsymbol{k}|=d+1\}, \quad K_{\boldsymbol{\sigma}}=\{\boldsymbol{k}: \boldsymbol{\sigma} \leq \boldsymbol{k} \text { and }|\boldsymbol{k}|=d+1\} .
$$


The family $\Pi_{\omega}^{R}$ is defined by $A=\left\{\boldsymbol{\alpha} \in \mathbb{N}^{n}: \boldsymbol{\alpha} \leq \boldsymbol{d}\right\}, \psi_{\omega}$ as in (34) for a fixed $\hat{\psi} \in \mathcal{C}_{0}^{\boldsymbol{d}}([\mathbf{0}, \mathbf{1}])$ and satisfy $\mathrm{H}_{\Pi}$ according to 48 with

$$
\begin{aligned}
& \boldsymbol{\gamma}=-\boldsymbol{d}-\mathbf{1} / q \\
& K_{\mathbf{0}}=\left\{\left(0, \ldots, d_{i}+1, \ldots, 0\right): i=1, \ldots, n\right\} \\
& K_{\boldsymbol{\sigma}}=\left\{\left(\sigma_{1}, \ldots, d_{i}+1, \ldots, \sigma_{n}\right): i=1, \ldots, n\right\}
\end{aligned}
$$

A graphical representation of the index sets for $\Pi_{\omega}^{S}$ and $\Pi_{\omega}^{R}$ is shown in Fig. 8 and 9. Remembering Propositions $3, \mathrm{H}_{\mathcal{M}}$ is implied by different mesh properties depending on whether $p \leq q$ or $p>q$. This leads to the following theorem.

Theorem 5 Suppose that $\mathrm{H}_{\mathbb{P}}, \mathrm{H}_{\lambda}, \mathrm{H}_{\phi}, \mathrm{H}_{\mathrm{s}}, \mathrm{H}_{\mathrm{E}}, \mathrm{H}_{\star}$ hold and $(1 / q, 1 / p) \in R_{d+1-|\boldsymbol{\sigma}|}$ for $\Pi_{\omega}^{S}$ and in $R_{\min (\boldsymbol{d}-\boldsymbol{\sigma})+1}$ for $\Pi_{\omega}^{R}$. Then (21), (22), (31) and (32) hold under the additional assumptions listed in the following table.

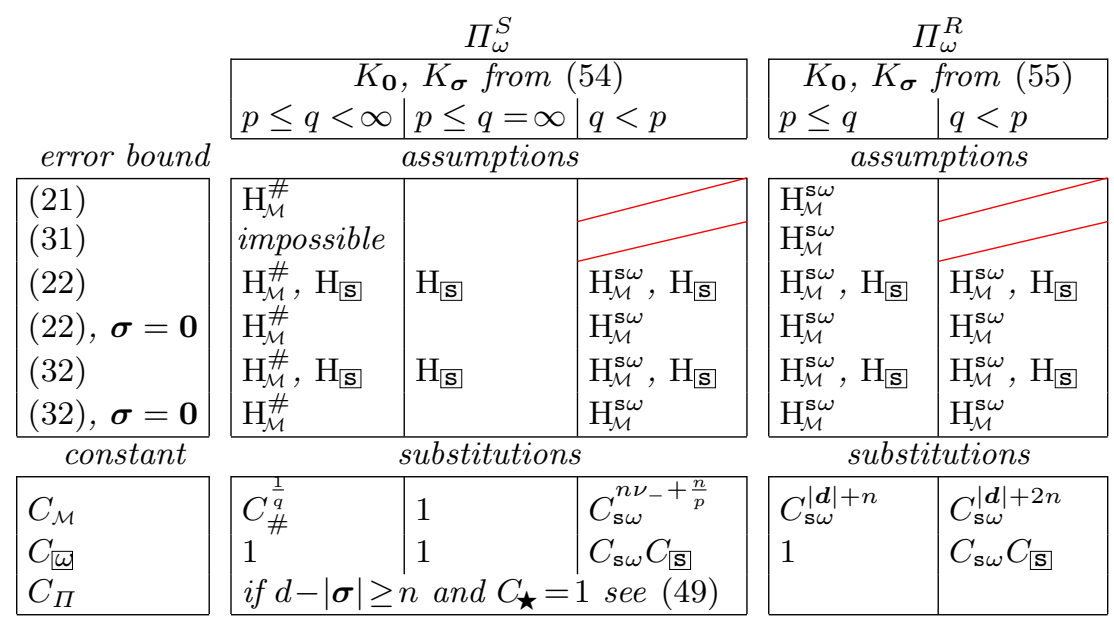

Empty cells mean the estimate holds without additional assumptions. A stroked cell means that the estimate does not apply and one should refer to Theorem 2. The table also lists possible substitutions for $C_{\mathcal{M}}, C_{\varpi}$ and $C_{\Pi}$.

Example 1 For $\boldsymbol{\sigma}=\mathbf{0}$ and $p=q=2$ one obtains 22 for $\Pi_{\omega}^{S}$ from $\mathrm{H}_{\mathbb{P}}, \mathrm{H}_{\lambda}$ $\mathrm{H}_{\phi}, \mathrm{H}_{\mathrm{s}}, \mathrm{H}_{\mathrm{E}}, \mathrm{H}_{\star}$ plus $\mathrm{H}_{\mathcal{M}}^{\#}$ with the substitution from the same column in $C$, i.e., $C_{\mathcal{M}}=C_{\#}^{1 / 2}, C_{\varpi}=1$ and $\# K_{\mathbf{0}}=\left(\begin{array}{c}d+n-1 \\ n-1\end{array}\right)$ giving

$$
C=4\left(\begin{array}{c}
d+n-1 \\
n-1
\end{array}\right)^{\frac{1}{2}} C_{\lambda} C_{\Phi} C_{\Pi} C_{\mathrm{s}}^{\frac{n}{2}} C_{\mathrm{E}} C_{\#}^{\frac{1}{2}}
$$

Remark 3 For $p>q$ element shape regularity, i.e. $\mathrm{H}_{[}$, is required indirectly as it is implied by the assumptions $\mathrm{H}_{\mathcal{M}}^{\mathrm{s \omega}}$ and $\mathrm{H}_{[\mathbf{S}}$, For $p \leq q$ element shape regularity is not required and shape regularity of the $\mathbf{s}_{\phi}$ 's is sufficient. Finally for $q=\infty$ and $\boldsymbol{\sigma}=\mathbf{0}$ no shape regularity assumption is required. 

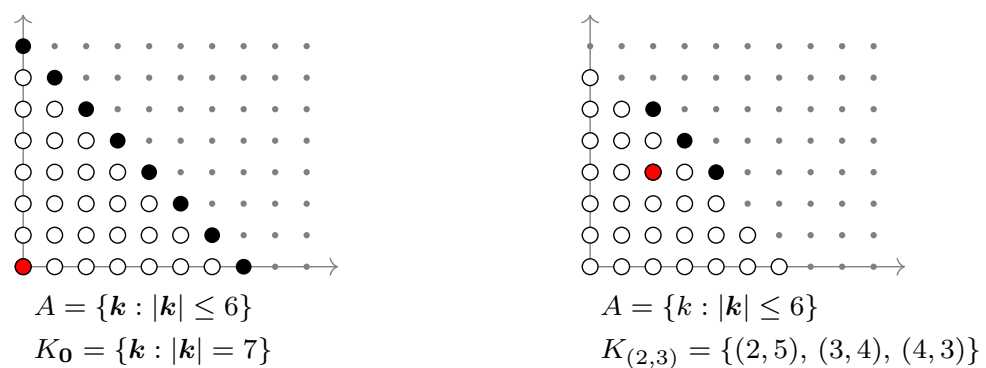

Fig. 8 Some examples of $A(0)$ and $K_{\boldsymbol{\beta}}(\bullet)$ for Sobolev seminorms and given $\boldsymbol{\beta}(\bullet)$.
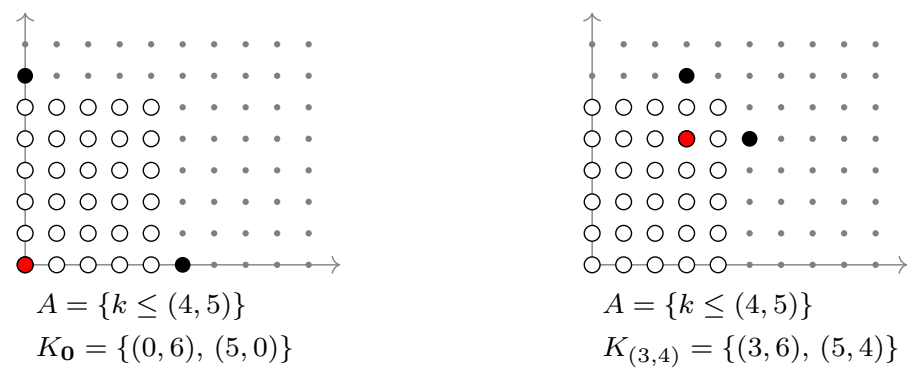

Fig. 9 Examples of $A(\circ)$ and $K_{\boldsymbol{\beta}}(\bullet)$ for reduced seminorms and given $\boldsymbol{\beta}(\bullet)$.

\section{Analytic functions and exponential convergence}

In this section we consider a sequence of spline spaces $\left\{\mathbb{S}_{d}=\operatorname{span} \Phi_{d}\right\}_{d \geq|\boldsymbol{\sigma}|+n}$ of degree $\boldsymbol{d}=(d, \ldots, d)$ and defined on the same domain $\Omega$. To each space we associate a corresponding operator $\aleph_{d}$ and we study the behavior of $\left\|\partial^{\sigma}\left(f-\aleph_{d} f\right)\right\|_{p}$ as a function of $d$.

\subsection{Exponential convergence}

The following theorem implies that the error decreases exponentially as the degree increases, provided that the space resolution is sufficiently small.

Theorem 6 Let $\left\{\Phi_{d}\right\},\left\{\aleph_{d}\right\}, d \geq|\boldsymbol{\sigma}|+n$ be a sequence of generating systems such that $\mathbf{s}_{\phi}$ is a box for all $\phi \in \bigcup_{d} \Phi_{d}$. Suppose $\mathrm{H}_{\mathbb{P}}, \mathrm{H}_{\lambda}, \mathrm{H}_{\phi}, \mathrm{H}_{\mathrm{s}}, \mathrm{H}_{\mathrm{E}} \mid \mathrm{H}_{[\mathbf{s}}$ hold with constants satisfying

$$
C_{\lambda} C_{\Phi} C_{\mathbf{s}}^{|\boldsymbol{\sigma}|} C_{\mathrm{E}} C_{[\mathbf{S}]}^{|\boldsymbol{\sigma}|} \leq C_{e} B_{e}^{d+1}(d+1)^{S_{e}}
$$

for some $C_{e}, B_{e}, S_{e}>0$. Then for all analytic function $f: \Omega \subseteq \mathbb{R}^{n} \rightarrow \mathbb{R}$ and $\Delta$ and $\boldsymbol{r}$ as in Proposition 2 we have

$$
\left\|\partial^{\sigma}\left(f-\aleph_{d} f\right)\right\|_{\infty} \leq C_{\boldsymbol{\sigma}}(d+1)^{n+S_{e}+|\boldsymbol{\sigma}|} \hat{\tau}^{d+1-|\boldsymbol{\sigma}|}\|f\|_{\infty, \Delta}
$$

where

$$
\hat{\tau}:=2 n^{\frac{3}{2}} B_{e} \sup _{d}\left\|\frac{\max \mathrm{h}_{\Phi_{d}}}{\min \boldsymbol{r}}\right\|_{\infty}, \quad C_{\boldsymbol{\sigma}}:=4 C_{e}\left(2 n^{\frac{3}{2}} B_{e}\right)^{|\boldsymbol{\sigma}|}\left\|(\min \boldsymbol{r})^{-|\boldsymbol{\sigma}|}\right\|_{\infty} .
$$


Proof Since the $\mathbf{s}_{\phi}$ are boxes, $\mathrm{H}_{\star}$ holds with $C_{\star}=1$. Theorem 5 applies and 32 holds with $K_{\mathbf{0}}, K_{\boldsymbol{\sigma}}$ as in (54) for $p=q=\infty$. Bounding $\left|\partial^{\boldsymbol{k}} f(\boldsymbol{x})\right|$ as in Proposition 1 and noting $K_{\mathbf{0}} \cup K_{\boldsymbol{\sigma}}=K_{\mathbf{0}}$ we have

$$
\left\|\partial^{\boldsymbol{\sigma}}\left(f-\aleph_{d} f\right)\right\|_{\infty} \leq C \# K_{\mathbf{0}} \max _{\substack{\phi \in \Phi_{d} \\|\boldsymbol{k}|=d+1}}\left\{\left\|\frac{\boldsymbol{k} !\left(\max \boldsymbol{h}_{\mathbf{s}_{\phi}}\right)^{d+1-|\boldsymbol{\sigma}|}}{\boldsymbol{r}^{\boldsymbol{k}}}\right\|_{\infty, \mathbf{s}_{\phi}}\right\}\|f\|_{\infty, \Delta}
$$

where using the substitutions from Theorem $5, C_{\Pi}$ from 49 and 56

$$
C=2 C_{\lambda} C_{\Phi} C_{\Pi} C_{\mathbf{s}}^{|\boldsymbol{\sigma}|} C_{\mathrm{E}} C_{\mathcal{M}}\left(C_{[\boldsymbol{\omega}}^{\left|\boldsymbol{\nu}_{-}\right|}+C_{\left[\mathbf{S}|+| \boldsymbol{\nu}_{-} \mid\right.}^{\mid \boldsymbol{|}}\right) \leq 4 C_{e} \frac{(d+1)^{S_{e}}\left(2 n^{\frac{3}{2}} B_{e}\right)^{d+1}}{(d-|\boldsymbol{\sigma}|) !} .
$$

The result follows using $\# K_{\mathbf{0}} \leq(d+1)^{n-1}, \boldsymbol{k} ! /(d-|\boldsymbol{\sigma}|) ! \leq(d+1)^{|\boldsymbol{\sigma}|+1}$ and

$$
\frac{\left(\max \boldsymbol{h}_{\mathbf{s}_{\phi}}\right)^{d+1-|\boldsymbol{\sigma}|}}{\boldsymbol{r}^{\boldsymbol{k}}} \leq \frac{1}{(\min \boldsymbol{r})^{|\boldsymbol{\sigma}|}} \frac{\operatorname{maxh}_{\Phi_{d}}^{d+1-|\boldsymbol{\sigma}|}}{(\min \boldsymbol{r})^{d+1-|\boldsymbol{\sigma}|}} \leq \frac{1}{(\min \boldsymbol{r})^{|\boldsymbol{\sigma}|}} \frac{\hat{\tau}^{d+1-|\boldsymbol{\sigma}|}}{\left(2 n^{\frac{3}{2}} B_{e}\right)^{d+1-|\boldsymbol{\sigma}|}} .
$$

If $\hat{\tau}<1$ then $(d+1)^{n+S_{e}+|\boldsymbol{\sigma}|} \hat{\tau}^{d+1-|\boldsymbol{\sigma}|}$ decreases exponentially in $d$. More precisely for all $1>\tau>\hat{\tau}$ we have

$$
\left\|\partial^{\sigma}\left(f-\aleph_{d} f\right)\right\|_{\infty} \leq C_{\tau} \tau^{d+1-|\sigma|}\|f\|_{\infty, \Delta}
$$

where $C_{\tau}:=C_{\boldsymbol{\sigma}} \max _{d \geq|\boldsymbol{\sigma}|+n}\left\{(d+1)^{n+S_{e}+|\boldsymbol{\sigma}|}(\hat{\tau} / \tau)^{d+1-|\boldsymbol{\sigma}|}\right\}$ is bounded independently of $d$. This shows exponential convergence.

Note that by $h$-refinement of the $\left\{\Phi_{d}\right\}$ it is always possible to obtain $\hat{\tau}<1$. We are able to prove exponential convergence on the space dimension by linking the space resolution with the cardinality of $\Phi$ as done in the following proposition.

Proposition 9 If $\operatorname{span} \Phi \supseteq \mathbb{P}_{\boldsymbol{d}}$ and the $\mathbf{s}_{\phi}$ are boxes then

$$
(\boldsymbol{d}+\mathbf{1})^{\mathbf{1}} \int_{\Omega} \mathrm{h}_{\Phi}^{-1} d \boldsymbol{x} \leq \# \Phi \leq C_{g} C_{\mathrm{E}} \int_{\Omega} \mathrm{h}_{\Phi}^{-1} d \boldsymbol{x}
$$

where the upper bound requires $\mathrm{H}_{\mathrm{E}}$ and that for all $\omega \in \mathcal{M}$

$$
\frac{\max \left\{\boldsymbol{h}_{\mathbf{s}_{\phi}}^{\mathbf{1}}: \phi \in \mathrm{A}_{\omega}\right\}}{\min \left\{\boldsymbol{h}_{\mathbf{s}_{\phi}}^{\mathbf{1}}: \phi \in \mathrm{A}_{\omega}\right\}} \leq C_{g}
$$

Proof First we rewrite $\# \Phi$ as a sum of integrals

$$
\# \Phi=\sum_{\phi \in \Phi} 1=\sum_{\phi \in \Phi} \int_{\mathbf{s}_{\phi}} \boldsymbol{h}_{\mathbf{s}_{\phi}}^{-1} d \boldsymbol{x}=\sum_{\phi \in \Phi} \sum_{\omega \subseteq \mathbf{s}_{\phi}} \int_{\omega} \boldsymbol{h}_{\mathbf{s}_{\phi}}^{-1} d \boldsymbol{x}=\sum_{\omega \in \mathcal{M}} \int_{\omega} \sum_{\phi \in \mathrm{E}_{\omega}} \boldsymbol{h}_{\mathbf{s}_{\phi}}^{-1} d \boldsymbol{x} .
$$

Since $\# \mathrm{E}_{\omega} \geq(\boldsymbol{d}+\mathbf{1})^{\mathbf{1}}$ and $\boldsymbol{h}_{\mathbf{s}_{\phi}}^{-\mathbf{1}} \geq \mathrm{h}_{\Phi}^{-\mathbf{1}}$ we have the lower bound. Using $\mathrm{H}_{\mathrm{E}}$ and 58 we have $\# \mathrm{E}_{\omega} \leq C_{\mathrm{E}}$ and $\boldsymbol{h}_{\mathrm{s}_{\phi}}^{-1} \leq C_{g} \mathrm{~h}_{\Phi}^{-1}$ from which the upper bound follows.

We now show exponential convergence as a function of the space dimension provided that space resolution is small, but not too small. 
Corollary 3 Under the assumptions of Theorem [6] and of Proposition 9, if $\hat{\tau}<1$ and there are $a, b>0$ such that for all $d \geq|\boldsymbol{\sigma}|+n$

$$
C_{\mathrm{E}} C_{g} \leq a(d+1)^{n}, \quad \frac{1}{b} \frac{\hat{\tau} \min \boldsymbol{r}}{2 n^{\frac{3}{2}} B_{e}} \leq \operatorname{minh}_{\Phi_{d}}, \forall \boldsymbol{x} \in \Omega,
$$

then there is $\tau_{\#}<1$ and $C_{\tau_{\#}}$ independent of $d$ and $\Phi_{d}$ such that

$$
\left\|\partial^{\sigma}\left(f-\aleph_{d} f\right)\right\|_{\infty} \leq C_{\tau_{\#}} \tau_{\#}^{\left(\# \Phi_{d}\right)^{1 / n}}\|f\|_{\infty, \Delta} .
$$

Proof From (59) we have

$$
\mathrm{h}_{\Phi_{d}}^{-1} \leq\left(\min \mathrm{h}_{\Phi_{d}}\right)^{-n} \leq b^{n} \hat{\tau}^{-n}\left(2 n^{\frac{3}{2}} B\right)^{n}(\min \boldsymbol{r})^{-n} .
$$

Proposition 9 gives

$$
\# \Phi_{d} \leq a(d+1)^{n} \int_{\Omega} \mathrm{h}_{\Phi_{d}}^{-1} d x \leq t^{-n}(d+1)^{n}
$$

where $t^{-n}:=a b^{n} \hat{\tau}^{-n}\left(2 n^{\frac{3}{2}} B_{e}\right)^{n} \int_{\Omega}(\min \boldsymbol{r}(\boldsymbol{x}))^{-n} d \boldsymbol{x}$. From the above we deduce $d+1 \geq t\left(\# \Phi_{d}\right)^{\frac{1}{n}}$. By $(57)$, and setting $\tau_{\#}:=\tau^{t}$ and $C_{\tau_{\#}}:=C_{\tau} \tau^{-|\sigma|}$, we have the result for all $1>\tau_{\#}>\hat{\tau}^{t}$.

\section{B-splines and coefficient functionals}

Many of the spline spaces used in applications are generated by B-splines and in Section 8 we consider tensor product splines (TPS), analysis suitable T-splines (AST), hierarchical splines (HS) and the locally refined splines (LR). This section recalls some properties of B-splines and TPS that help in the construction of $\aleph$ satisfying the assumptions in Sections 3...6. For more material and proofs of some of the results below we refer to [7] 26] 36.

We recall first the properties of B-splines that we need for $\mathrm{H}_{\mathbb{P}}$ and $\mathrm{H}_{\phi}$. Then we introduce two families of coefficient functionals for TPS that satisfy $\mathrm{H}_{\lambda}$. The functional $S_{\phi}$ from 34 provides the smallest bound for $C_{\lambda}$ available in the literature, but is restricted to $\operatorname{supp} S_{\phi}=\operatorname{supp} \phi$. Another functional $G_{\phi, \eta}$ allows for the choice of $\eta=\operatorname{supp} G_{\phi, \eta} \subseteq \operatorname{supp} \phi$ and it is based on [25].

7.1 B-splines and their smoothness: $\mathrm{H}_{\phi}$

A univariate B-spline of degree $d$ is a compactly supported non-negative piecewise polynomial with minimal support. Each B-spline of degree $d$ is uniquely associated to a knot vector, a non-decreasing sequence of $d+2$ real numbers that encodes its smoothness properties and its polynomial subdomains. The univariate B-spline $\varphi$ of degree $d$ associated with the knot vector $\Theta(\varphi):=\left[\theta_{1}(\varphi), \ldots, \theta_{d+2}(\varphi)\right]$ has support $\left[\theta_{1}(\varphi), \theta_{d+2}(\varphi)\right]$ so that $h_{\varphi}=\theta_{d+2}(\varphi)-\theta_{1}(\varphi)$ and it is defined by

$$
\varphi(x):=h_{\varphi}\left[\theta_{1}(\varphi), \ldots, \theta_{d+2}(\varphi)\right](\cdot-x)_{+}^{d},
$$

where $\left[\theta_{1}, \ldots, \theta_{d+2}\right] f$ is the usual divided difference of $f$, that in this case is given by $f(\theta):=(\theta-x)_{+}^{d}$. 
We recall that $\left.\varphi\right|_{Z} \in \mathbb{P}_{d}$ for all $Z$ of the form $\left[\theta_{i}(\varphi), \theta_{i+1}(\varphi)\right]$ with $\theta_{i}(\varphi)<$ $\theta_{i+1}(\varphi)$. If $m_{\Theta_{\varphi}}(x)$ is the number of repetitions of $x$ in $\Theta(\varphi)$ then $\varphi$ has $d-m_{\Theta_{\varphi}}(x)$ continuous derivatives at $x$.

Note that from 60 and properties of divided differences it follows that

$$
\int \varphi(x) d x=h_{\varphi}\left[\theta_{1}(\varphi), \ldots, \theta_{d+2}(\varphi)\right] \int_{\operatorname{supp} \varphi}(\cdot-x)_{+}^{d} d x=\frac{h_{\varphi}}{d+1} .
$$

A $n$-variate tensor product B-spline $\phi$ of degree $\boldsymbol{d}=\left(d_{1}, \ldots, d_{n}\right)$ is a product of $n$ univariate B-splines

$$
\phi(\boldsymbol{x}):=\varphi_{1}\left(x_{1}\right) \cdots \varphi_{n}\left(x_{n}\right)
$$

and is defined by an $n$-tuple $\Theta(\phi):=\left(\Theta\left(\varphi_{1}\right), \ldots, \Theta\left(\varphi_{n}\right)\right)$ of knot vectors. We recall that $\boldsymbol{h}_{\phi}=\boldsymbol{h}_{\operatorname{supp} \phi}$.

Lemma 3 For all B-spline $\phi=\varphi_{1} \cdots \varphi_{n}$ of degree $\boldsymbol{d}$ we have

$$
\frac{\boldsymbol{h}_{\phi}^{\mathbf{1} / p}}{(\boldsymbol{d}+\mathbf{1})^{\mathbf{1}}} \leq\|\phi\|_{p} \leq \frac{\boldsymbol{h}_{\phi}^{\mathbf{1 / p}}}{(\boldsymbol{d}+\mathbf{1})^{\mathbf{1 / p}}}, \quad 1 \leq p \leq \infty .
$$

Proof By 62 we have $\|\phi\|_{p}=\left\|\varphi_{1}\right\|_{p} \cdots\left\|\varphi_{n}\right\|_{p}$. By 61) and Hölder's inequality

$$
1=\frac{d_{i}+1}{h_{\varphi_{i}}} \int_{\mathbb{R}} \varphi_{i}\left(x_{i}\right) d x_{i} \leq \frac{d_{i}+1}{h_{\varphi_{i}}}\left\|\varphi_{i}\right\|_{p} h_{\varphi_{i}}{ }^{1-1 / p}
$$

and the lower bound follows. Since $\left\|\varphi_{i}\right\|_{\infty} \leq 1$ we have

$$
\left(\int_{\mathbb{R}} \varphi_{i}\left(x_{i}\right)^{p} d x_{i}\right)^{1 / p} \leq\left(\int_{\mathbb{R}} \varphi_{i}\left(x_{i}\right) d x_{i}\right)^{1 / p}=\frac{h_{\varphi_{i}}^{1 / p}}{\left(d_{i}+1\right)^{1 / p}}
$$

giving the upper bound.

The $L^{\infty}$ norms of the derivatives of a B-spline depend on its knot vectors. For $\phi=\varphi_{1} \cdots \varphi_{n}$ let

$$
\Delta_{\phi, i, k}:=\min _{\ell=k+1, \ldots, d_{i}+2}\left\{\theta_{\ell}\left(\varphi_{i}\right)-\theta_{\ell-k}\left(\varphi_{i}\right)\right\}
$$

Then, as stated in the following proposition, $\mathrm{H}_{\phi}$ is implied by $\mathrm{H}_{\phi}^{\Theta} \frac{\Delta_{\phi, i, d_{i}+1}}{\Delta_{\phi, i, d_{i}-\sigma_{i}+1}} \leq C_{\Theta}$, for all $\phi \in \Phi, i=1, \ldots, n$, knot vector regularity

Proposition 10 Let $\Phi$ be a collection of B-splines of the same degree satisfying $\mathrm{H}_{\phi}^{\Theta}$. Then $\mathrm{H}_{\phi}$ holds with

$$
C_{\Phi}=\frac{(\boldsymbol{d}+\mathbf{1}) ! 2^{|\boldsymbol{\sigma}|}}{(\boldsymbol{d}-\boldsymbol{\sigma}) !} C_{\Theta}^{|\boldsymbol{\sigma}|} .
$$


Proof Using $\partial^{\sigma} \phi(\boldsymbol{x})=\partial_{1}^{\sigma_{1}} \varphi_{1}\left(x_{1}\right) \cdots \partial_{n}^{\sigma_{n}} \varphi_{n}\left(x_{n}\right), \mathrm{H}_{\phi}^{\Theta}$, and the following univariate bound [36, Theorem 4.22]

$$
\left\|\partial^{\sigma_{i}} \varphi_{i}\right\|_{\infty} \leq \frac{d_{i} ! 2^{\sigma_{i}}}{\left(d_{i}-\sigma_{i}\right) !} \prod_{k=d_{i}+1-\sigma_{i}}^{d} \Delta_{\phi, i, k}^{-1} \leq \frac{d_{i} ! 2^{\sigma_{i}}}{\left(d_{i}-\sigma_{i}\right) !} \Delta_{\phi, i, d_{i}+1-\sigma_{i}}^{-\sigma_{i}}
$$

we have

$$
\left\|\partial^{\boldsymbol{\sigma}} \phi\right\|_{\infty} \leq \frac{\boldsymbol{d} ! 2^{|\boldsymbol{\sigma}|}}{(\boldsymbol{d}-\boldsymbol{\sigma}) !} \prod_{i=1}^{n} \Delta_{\phi, i, d_{i}+1-\sigma_{i}}^{-\sigma_{i}} \leq \frac{\boldsymbol{d} ! 2^{|\boldsymbol{\sigma}|}}{(\boldsymbol{d}-\boldsymbol{\sigma}) !} C_{\Theta}^{|\boldsymbol{\sigma}|} \boldsymbol{h}_{\phi}^{-\boldsymbol{\sigma}} .
$$

$\mathrm{H}_{\phi}$ follows using Hölder's inequality and the lower bound for $\|\phi\|_{p}$ in 63 .

7.2 Tensor product splines and polynomial representation

A $(d+1)$-open knot vector is a non-decreasing sequence of real numbers $\Xi:=$ $\left[\xi_{1}, \ldots, \xi_{s+d+1}\right], s \geq d+1$, with the following properties

$$
\xi_{1}=\cdots=\xi_{d+1}, \quad \xi_{s+1}=\cdots=\xi_{s+d+1}, \quad \xi_{i+d+1}>\xi_{i}, i=1, \ldots, s .
$$

Associated with $\Xi$ is the space $\mathbb{S}_{\Xi, d} \supseteq \mathbb{P}_{d}$ of piecewise polynomials of degree $d$ on the mesh $\left\{\left[\xi_{i}, \xi_{i+1}\right]: \xi_{i}<\xi_{i+1}\right\}$ that have $d-m_{\Xi}(x)$ continuous derivatives at $x \in\left[\xi_{1}, \xi_{s+d+1}\right]$, where $m_{\Xi}(x)$ is the number of repetitions of $x$ in $\Xi$. The B-splines $\left\{\varphi_{1}, \ldots, \varphi_{s}\right\}$, where $\Theta\left(\varphi_{i}\right)=\left[\xi_{i}, \ldots, \xi_{i+d+1}\right]$, constitute a basis of $\mathbb{S}_{\Xi, d}$ and provide a non-negative partition of unity.

Similarly, with an $n$-tuple of knot vectors $\Xi:=\left(\Xi_{1}, \ldots, \Xi_{n}\right)$ where each $\Xi_{i}:=$ $\left[\xi_{i, 1}, \ldots, \xi_{i, s_{i}+d_{i}+1}\right]$ is a $\left(d_{i}+1\right)$-open knot vector we associate the tensor product spline space

$$
\mathbb{S}_{\Xi, \boldsymbol{d}}:=\mathbb{S}_{\Xi_{1}, d_{1}} \otimes \cdots \otimes \mathbb{S}_{\Xi_{n}, d_{n}}
$$

The canonical basis has $\boldsymbol{s}^{\mathbf{1}}$ elements $\phi_{\boldsymbol{i}}$, with $\boldsymbol{i} \in[\mathbf{1}, \boldsymbol{s}] \cap \mathbb{N}^{n}$

$$
\phi_{\boldsymbol{i}}(\boldsymbol{x}):=\varphi_{1, i_{1}}\left(x_{1}\right) \cdots \varphi_{n, i_{n}}\left(x_{n}\right), \quad \Theta\left(\varphi_{j, i_{j}}\right):=\left[\xi_{j, i_{j}}, \ldots, \xi_{j, i_{j}+d_{j}+1}\right] .
$$

Any $g \in \mathbb{P}_{\boldsymbol{d}}$ can be written explicitly as linear combination of the $\phi_{\boldsymbol{i}}$

$$
g=\sum_{i \in[\mathbf{1}, \boldsymbol{s}]} \mathfrak{c}\left(g, \phi_{\boldsymbol{i}}\right) \phi_{\boldsymbol{i}}
$$

The coefficients $\mathfrak{c}\left(g, \phi_{\boldsymbol{i}}\right)$ are sometimes called blossoms and depend only on $g$ and the internal knots $\xi_{j, i_{j}+1}, \ldots, \xi_{j, i_{j}+d_{j}}, j=1, \ldots, n$ of $\phi_{i}$. 
7.3 Two families of coefficient functionals

A collection $\left\{S_{\varphi}\right\}$ of bi-orthogonal functionals for the B-spline basis of $\mathbb{S}_{\Xi, d}$ was constructed in 34 . The collection satisfies $\mathrm{H}_{\lambda}$ with $C_{\lambda}=(d+1) 2^{d+1}$. No collection of bi-orthogonal functionals can satisfy $\left[\mathrm{H}_{\lambda}\right.$ with $C_{\lambda}<k_{d, p}$ where $k_{d, p}$ is the condition number of the B-spline basis. It is known, see [36, p.528] for references, that $k_{d, p} \geq c(d+1)^{-1 / p} 2^{d+1}$ for some $c>0$ independent of $d$ and $p$. In this sense the functionals $S_{\varphi}$ 's are close to optimal.

The functional in 34] is defined only for univariate B-splines, but it can easily be extended to the tensor product B-splines. To a B-spline $\phi=\varphi_{1} \cdots \varphi_{n}$ we associate the operator $S_{\phi}$ defined by

$$
S_{\phi}(f):=\int_{\operatorname{supp} \phi} w_{\varphi_{1}}\left(x_{1}\right) \cdots w_{\varphi_{n}}\left(x_{n}\right) f(\boldsymbol{x}) d \boldsymbol{x},
$$

where the $w_{\varphi_{i}}$ are described in [34] and have the same support as $\varphi_{i}$.

The bi-orthogonality properties of the $S_{\phi}$, their support and their norm directly lead to the following proposition.

Proposition 11 Let $\Phi$ be a collection of B-splines of degree $\boldsymbol{d}$, then $\Lambda=\left\{S_{\phi}\right.$ : $\phi \in \Phi\}$ satisfies the assumptions $\mathrm{H}_{\lambda}$ and $\mathrm{H}_{\mathbf{s}}$ with $C_{\lambda}=(\boldsymbol{d}+\mathbf{1})^{\mathbf{1}} 2^{|\boldsymbol{d}|+n}$ and $C_{\mathbf{s}}=1$. Moreover, if $\Phi$ is a TPS basis satisfying $\mathrm{H}_{\phi}^{\ominus}$ then $\aleph$ defined by (6) is a projector onto span $\Phi$, and $\mathrm{H}_{\mathbb{P}}, \mathrm{H}_{\lambda}, \mathrm{H}_{\phi}$ and $\mathrm{H}_{\mathrm{s}}$ are satisfied.

We will use the functional $S_{\phi}$ for TPS, AST, THB and a subclass of LR. In the next subsection and for general LR we need a functional with a smaller support. We obtain it by modifying the construction in 25$]$.

Definition 2 Let $\phi=\varphi_{1} \cdots \varphi_{n}$ be a B-spline of degree $\boldsymbol{d}$ and $\eta=\eta_{1} \times \cdots \times \eta_{n} \subseteq$ $\operatorname{supp} \phi$ be a box. We define

$$
G_{\phi, \eta}(f):=\frac{1}{\mu(\eta)} \int_{\eta} w_{\varphi_{1}, \eta_{1}}\left(x_{1}\right) \cdots w_{\varphi_{n}, \eta_{n}}\left(x_{n}\right) f(\boldsymbol{x}) d \boldsymbol{x}
$$

where $w_{\varphi_{i}, \eta_{i}} \in \mathbb{P}_{d_{i}}$ are such that

$$
G_{\phi, \eta}(g)=\mathfrak{c}(g, \phi), \quad \forall g \in \mathbb{P}_{\boldsymbol{d}}
$$

Proposition 12 For all $\phi$ and $\eta \subseteq \operatorname{supp} \phi, G_{\phi, \eta}$ is well defined. If $\Phi$ is a collection of B-splines of degree $\boldsymbol{d}$, then for $\Lambda=\left\{G_{\phi, \eta_{\phi}}: \eta_{\phi} \subseteq \operatorname{supp} \phi, \phi \in \Phi\right\}$ the assumptions $\mathrm{H}_{\lambda}$ and $\mathrm{H}_{\mathrm{s}}$ hold with constants $C_{\mathrm{s}}=1$ and

$$
C_{\lambda}=(\boldsymbol{d}+\mathbf{1})^{\mathbf{1}-\mathbf{1} / p} \max _{\phi \in \Phi}\left\{\boldsymbol{h}_{\phi}^{\boldsymbol{d}+\mathbf{1} / p} \boldsymbol{h}_{\eta_{\phi}}^{-\boldsymbol{d}-\mathbf{1} / p}\right\} \prod_{i=1}^{n}\left\|H_{d_{i}}^{-1}\right\| .
$$

Here $H_{d}$ is the Hilbert matrix of order $d+1$, i.e., the element in position $(s, t)$ is $(s+t-1)^{-1}$, and $\|\cdot\|$ is the $\ell^{\infty}$ operator norm. Moreover, if $\Phi$ is a TPS basis satisfying $\mathrm{H}_{\phi}^{\Theta}$ then with $\aleph$ defined by $6,, \mathrm{H}_{\mathbb{P}}, \mathrm{H}_{\lambda}, \mathrm{H}_{\phi}$ and $\mathrm{H}_{\mathrm{s}}$ are satisfied. If additionally each $\eta_{\phi}$ is contained in some $\omega_{\phi} \in \mathcal{M}$, then $\aleph$ is a projector onto $\operatorname{span} \Phi$. 
Proof Due to the tensor product structure, all claims follow from the univariate case. Let $\varphi$ be a B-spline of degree $d$ and $\eta \subseteq \operatorname{supp} \varphi$ be the desired support.

First we show that $w_{\varphi, \eta}$ is determined by 70 . Let $t:=\frac{x-\min \eta}{\mu(\eta)}$, then expressing $w_{\varphi, \eta}(x)$ as

$$
w_{\varphi, \eta}(x):=\sum_{j=0}^{d} c_{j} t(x)^{j}
$$

we find

$$
G_{\varphi, \eta}\left(t^{i}\right)=\sum_{j=0}^{d} c_{j} \frac{1}{\mu(\eta)} \int_{\eta} t(x)^{i+j} d x=\sum_{j=0}^{d} \frac{c_{j}}{i+j+1} .
$$

Thus 70 is equivalent to $c:=\left(c_{1}, \ldots, c_{d+1}\right)$ satisfying

$$
H_{d} \boldsymbol{c}=\boldsymbol{b},
$$

where $\boldsymbol{b}:=\left(\mathfrak{c}\left(t^{0}, \varphi\right), \ldots, \mathfrak{c}\left(t^{d}, \varphi\right)\right)$. Since $H_{d}$ is square and invertible the system is well posed and $w_{\varphi, \eta}$ is uniquely determined.

We now prove $\mathrm{H}_{\lambda}$. By Hölder's inequality, and $0 \leq t(x) \leq 1$ for $x \in \eta$, we have

$$
\left\|G_{\varphi, \eta}\right\|_{* p} \leq \frac{\left\|w_{\varphi, \eta}\right\|_{p^{\prime}, \eta}}{\mu(\eta)} \leq \frac{\left\|w_{\varphi, \eta}\right\|_{\infty, \eta}}{\mu(\eta)^{1 / p}} \leq \frac{(d+1)}{\mu(\eta)^{1 / p}}\left\|H_{d}^{-1}\right\|\|\boldsymbol{b}\|_{\ell^{\infty}} .
$$

To bound $\|\boldsymbol{b}\|_{\ell^{\infty}}$ we use Marsden's identity

$$
\mathfrak{c}\left((y-x)^{d}, \phi\right)=\prod_{i=2}^{d+1}\left(y-\theta_{i}(\phi)\right)
$$

to obtain

$$
\left|\mathfrak{c}\left(t^{r}, \phi\right)\right| \leq \frac{1}{(d-r) !} \frac{\mu(\operatorname{supp} \varphi)^{r}}{\mu(\eta)^{r}} \leq \frac{\mu(\operatorname{supp} \varphi)^{d}}{\mu(\eta)^{d}}, \quad r=0, \ldots, d
$$

Now $\mathrm{H}_{\lambda}$ for univariate B-splines follows from $(72)$ and $(63)$.

Finally if $\Phi$ is a TPS basis and $\eta_{\phi} \subseteq \omega_{\phi} \in \mathcal{M}$ then by (67) we have $G_{\phi, \eta_{\phi}}(\psi)=$ $\mathfrak{c}\left(\left.\psi\right|_{\omega_{\phi}}, \phi\right)=\delta_{\phi, \psi}$ for all $\psi \in \Phi$, and consequently $\aleph$ is a projector.

\section{Space specific results}

In this section we describe a specific approximation operator $\aleph$ for TPS, AST, THB and LR splines. For each operator we show which of the abstract assumptions of Section 3 hold and give an upper bound for the corresponding constants. Combining this information with Theorem 5 one obtains error bounds with Sobolev or reduced seminorms. 
8.1 Application to tensor product B-splines

Assuming the usual mesh regularity, i.e. $\mathrm{H}_{\mathcal{M}}^{\mathrm{s \omega}}$ and $\mathrm{H}_{[\mathbf{s}}$, we obtain all the error bounds in Theorem 5 for TPS. Compared to what can be found in the literature, see for example [27] [5] 6], we take into account the local mesh size for all combinations of $p$ and $q$.

Theorem 7 Let $\Phi$ be a TPS basis satisfying $\mathrm{H}_{\phi}^{\Theta}$. Then the operator $\aleph$ corresponding to $\Lambda=\left\{\lambda_{\phi}=S_{\phi}: \phi \in \Phi\right\}$ is a projector onto $\mathbb{S}=\operatorname{span} \Phi$ and the assumptions $\mathrm{H}_{\mathbb{P}}, \mathrm{H}_{\lambda}, \mathrm{H}_{\phi}, \mathrm{H}_{\mathrm{s}}, \mathrm{H}_{\mathrm{E}}, \mathrm{H}_{\mathcal{M}}^{\#}$ and $\mathrm{H}_{\star}$ are satisfied with

$$
\begin{array}{lll}
C_{\lambda}=(\boldsymbol{d}+\mathbf{1})^{\mathbf{1}} 2^{|\boldsymbol{d}|+n}, & C_{\Phi}=\frac{(\boldsymbol{d}+\mathbf{1}) ! 2^{|\boldsymbol{\sigma}|}}{(\boldsymbol{d}-\boldsymbol{\sigma}) !} C_{\Theta}^{|\boldsymbol{\sigma}|}, & C_{\mathrm{s}}=1, \\
C_{\mathrm{E}}=(\boldsymbol{d}+\mathbf{1})^{\mathbf{1}}, & C_{\#}=(\boldsymbol{d}+\mathbf{1})^{\mathbf{1}}, & C_{\star}=1 .
\end{array}
$$

Proof For TPS $\mathrm{H}_{\mathcal{M}}^{\#}$ holds with $C_{\#}=(\boldsymbol{d}+\mathbf{1})^{\mathbf{1}}$, and since $\operatorname{supp} \phi=\mathbf{s}_{\phi}$ we have $\mathrm{H}_{\mathrm{E}}$ with $C_{\mathrm{E}}=(\boldsymbol{d}+\mathbf{1})^{1}$. Then the thesis follows from Proposition 10 , Proposition 11

\subsection{Application to AST-splines}

Cubic T-splines were introduced in 37] 38 for geometric modeling applications. The idea was to reduce the number of control points by replacing the control polygon of TPS with a T-mesh. Depending on $\boldsymbol{d}$, a tensor product B-spline is associated with each vertex, edge or element of the T-mesh. The T-spline space is spanned by these B-splines.

Analysis Suitable T-splines (AST) avoid linear dependencies by restricting the class of allowed T-meshes [4] 24] 3]. AST spaces can be constructed in 2D 30] and are also defined for 3D domains [29]. In particular AST are dual compatible, cf. [4] 3] and bi-orthogonal functionals for TPS are bi-orthogonal to AST. A characterization of the piecewise polynomials spanned by an AST basis is in [9].

By Proposition 10 and and the bi-orthogonality properties of $S_{\phi}$ we obtain the following theorem. Compared to the result for TPS there is no a priori bound for $C_{\#}$ which can be used as a measure of the mesh complexity.

Theorem 8 Let $\Phi$ be an AST basis satisfying $\mathrm{H}_{\phi}^{\Theta}$. The operator $\aleph$ corresponding to $\Lambda=\left\{\lambda_{\phi}=S_{\phi}: \phi \in \Phi\right\}$ is a projector onto $\mathbb{S}=\operatorname{span} \Phi$ and $\overline{\mathrm{H}_{\mathbb{P}}}, \mathrm{H}_{\lambda} \mid \mathrm{H}_{\phi}, \mathrm{H}_{\mathrm{s}}, \mathrm{H}_{\mathrm{E}}$ and $\mathrm{H}_{\star}$ hold with

$$
\begin{array}{lll}
C_{\lambda}=(\boldsymbol{d}+\mathbf{1})^{\mathbf{1}} 2^{|\boldsymbol{d}|+n}, & C_{\Phi}=\frac{(\boldsymbol{d}+1) ! 2^{|\boldsymbol{\sigma}|}}{(\boldsymbol{d}-\boldsymbol{\sigma}) !} C_{\Theta}^{|\boldsymbol{\sigma}|}, & \\
C_{\mathrm{s}}=1, & C_{\mathrm{E}}=(\boldsymbol{d}+\mathbf{1})^{\mathbf{1}}, & C_{\star}=1 .
\end{array}
$$

\subsection{Application to THB splines}

Hierarchical spline were introduced in [19 23]. Quasi-interpolants have been constructed in [39] 40] based on the preservation of coefficient property [21] of the truncated basis 20]. See 25] for a recent survey. 
Let $\Psi_{1}, \ldots, \Psi_{s}$ be a sequence of TPS bases that span nested spaces, i.e. $i<$ $j \Rightarrow \operatorname{span} \Psi_{i} \subset \operatorname{span} \Psi_{j}$ and $\Omega=\Omega_{1} \supseteq \cdots \supseteq \Omega_{s}=\emptyset$ a corresponding sequence of closed domains. The hierarchical basis $\mathbb{H}$ is as follows

$$
\mathbb{H}:=\bigcup_{i=1}^{s}\left\{\psi \in \Psi_{i}: \operatorname{supp} \psi \subseteq \Omega_{i} \text { and } \operatorname{supp} \psi \cap\left(\Omega_{i} \backslash \Omega_{i+1}\right) \neq \emptyset\right\}
$$

The associated box-mesh $\mathcal{M}$ contains a similar selection of elements from the tensor product meshes $\mathcal{M}_{1}, \ldots, \mathcal{M}_{s}$ corresponding to $\Psi_{1}, \ldots, \Psi_{s}$ :

$$
\mathcal{M}:=\bigcup_{i=1}^{s}\left\{\omega \in \mathcal{M}_{i}: \omega \in \Omega_{i} \backslash \Omega_{i+1}\right\} .
$$

The truncation operator $\mathrm{T}_{i}: \operatorname{span} \Psi_{i} \rightarrow \operatorname{span}\left\{\psi \in \Psi_{i}:\left.\psi\right|_{\Omega \backslash \Omega_{i}} \neq 0\right\}$ is defined as

$$
\mathrm{T}_{i}\left(\sum_{\psi \in \Psi_{i}} c_{\psi} \psi\right):=\sum_{\substack{\psi \in \Psi_{i}: \\\left.\psi\right|_{\Omega \backslash \Omega_{i}} \neq 0}} c_{\psi} \psi
$$

By recursive truncation one obtains the truncated basis

$$
\mathbb{H}^{\mathrm{T}}:=\left\{\mathrm{T}_{s} \cdots \mathrm{T}_{i+1} \psi: \psi \in \mathbb{H} \cap \Psi_{i}, i=1, \ldots, s\right\} .
$$

It is convenient to abbreviate $\mathrm{T}_{s} \cdots \mathrm{T}_{i+1} \psi$ with $\mathrm{T} \psi$ and to annotate the symbols referring to $\mathbb{H}^{\mathrm{T}}$ with a superscript $\mathrm{T}$ to distinguish them from those referring to $\mathbb{H}$. One of the advantages of the truncated basis is that the coefficients of a polynomial $g \in \mathbb{P}_{\boldsymbol{d}}$ are the same as for TPS

$$
g=\sum_{\phi \in \mathbb{H}} \mathfrak{c}(g, \phi) \mathrm{T} \phi
$$

We say that a HB basis is $k$-admissible if for each $\omega \in \mathcal{M}_{j} \cap \mathcal{M}, \mathrm{A}_{\omega}$ contains only functions $\phi \in \Psi_{i}$ for $j-k<i \leq j$. A THB basis is $k$-admissible if the corresponding HB basis is. See also [13] 14].

Theorem 9 Let $\Phi$ be a $k$-admissible $T H B$ basis satisfying $\left[\mathrm{H}_{\phi}\right.$, and a be such that $\boldsymbol{h}_{\phi} \leq \boldsymbol{h}_{\omega}$, for all $\phi \in \Psi_{i}$ and $\omega \subseteq \operatorname{supp} \phi$, such that $\omega \in \mathcal{M}_{i} \cap \mathcal{M}$. Then the operator $\aleph$ corresponding to $\Lambda=\left\{\lambda_{\mathrm{T} \phi}=S_{\phi}: \mathrm{T} \phi \in \Phi\right\}$ is a projector onto $\mathbb{S}=\operatorname{span} \Phi$, and $\overline{\mathrm{H}_{\mathbb{P}}}, \mathrm{H}_{\lambda}, \mathrm{H}_{\mathrm{s}}, \mathrm{H}_{\mathrm{E}}$ and $\overline{\mathrm{H}_{\star}}$ hold with

$$
C_{\lambda}=(\boldsymbol{d}+\mathbf{1})^{\mathbf{1}} 2^{|\boldsymbol{d}|+n}, \quad C_{\mathrm{s}}=a, \quad C_{\mathrm{E}}=k(\boldsymbol{d}+\mathbf{1})^{\mathbf{1}}, \quad C_{\star}=1 .
$$

Proof $\mathrm{H}_{\mathbb{P}}$ follows from 76 . Since $\|\mathrm{T} \phi\|_{p} \leq\|\phi\|_{p}$ we have $\mathrm{H}_{\lambda}$ with $C_{\lambda}=(\boldsymbol{d}+$ 1) ${ }^{\mathbf{1}} 2^{|\boldsymbol{d}|+n}$. Noting that $\mathbf{s}_{\mathrm{T} \phi}=\operatorname{supp} S_{\phi}=\operatorname{supp} \phi$ we have $\boldsymbol{h}_{\mathrm{S}_{\mathrm{T}}}=\boldsymbol{h}_{\phi} \leq a \boldsymbol{h}_{\omega} \leq a \boldsymbol{h}_{\mathrm{T} \phi}$ and $\mathrm{H}_{\mathrm{s}}$ follows with $C_{\mathrm{s}}=a$. As $\Phi$ is $k$-admissible and $\mathbf{s}_{\mathrm{T} \phi}=\operatorname{supp} \phi$ we have $\mathrm{E}_{\omega}^{\mathrm{T}}=\mathrm{A}_{\omega}$ and consequently $\# \mathrm{E}_{\omega}^{\mathrm{T}} \leq k(\boldsymbol{d}+\mathbf{1})^{\mathbf{1}}$. Since $\mathbf{s}_{\mathrm{T} \phi}$ is a box we have $C_{\star}=1$.

Note that THB require more assumptions than TPS and AST. Comparing with 39 40, we obtain global estimates with different $p, q$ norms that take into consideration the local mesh size, see Theorem 5. 

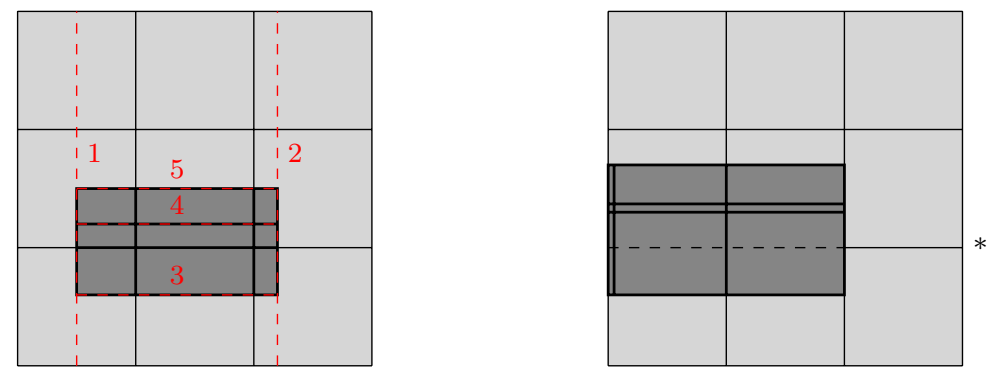

Fig. 10 Two examples with B-splines of degree $(2,2)$. On the left, $\phi(\square)$ is nested in $\psi(\square)$. The dashed red lines represent a possible sequence of knots insertions to obtain $\phi$ form $\psi$. On the right, $\phi$ is not nested in $\psi$ even if $\operatorname{supp} \phi \subseteq \operatorname{supp} \psi$ because the marked line corresponds to a knot of $\psi$, but not to a knot of $\phi$.

\subsection{Application to LR-splines}

LR-splines where introduced in [16], here we use the equivalent definition from 8. A box mesh $\mathcal{M}$ and a function $m$ that assigns a nonnegative integer to each interface between two elements define the spline space

$$
\mathbb{K}:=\left\{\begin{array}{rr}
f \in L^{\infty}(\Omega):\left.f\right|_{\omega} \in \mathbb{P}_{\boldsymbol{d}}, & \forall \omega \in \mathcal{M}, \\
f \in \mathcal{C}^{d_{i}-m(E)}(\stackrel{\circ}{E}), & \text { for all inter-element interfaces } E
\end{array}\right\},
$$

where $i$ is the direction of the normal to $E$. Here we assume that $\Omega$ is a box.

Given a B-spline $\phi=\varphi_{1} \cdots \varphi_{n}$ and $s \in\{1, \ldots, n\}$ we can insert a knot $\bar{\theta} \in$ $\left(\theta_{1}\left(\varphi_{s}\right), \theta_{d+2}\left(\varphi_{s}\right)\right)$ in $\Theta\left(\varphi_{s}\right)$ and obtain the two B-splines $\hat{\phi}=\varphi_{1} \cdots \hat{\varphi}_{s} \cdots \varphi_{n}$ and $\tilde{\phi}=\varphi_{1} \cdots \tilde{\varphi}_{s} \cdots \varphi_{n}$ whose knot vectors are $\Theta\left(\hat{\varphi}_{s}\right)=\left[\theta_{1}\left(\varphi_{s}\right), \ldots, \bar{\theta}, \ldots, \theta_{d+1}\left(\varphi_{s}\right)\right]$ and $\Theta\left(\tilde{\varphi}_{s}\right)=\left[\theta_{2}\left(\varphi_{s}\right), \ldots, \bar{\theta}, \ldots, \theta_{d+2}\left(\varphi_{s}\right)\right]$, respectively. We have

$$
\phi=a \hat{\phi}+b \tilde{\phi}, \quad a, b \in(0,1] .
$$

Knot insertion defines a partial ordering on the set of B-splines, we write $\phi \prec \psi$, if there exists a sequence of B-splines $\phi_{1}, \ldots, \phi_{r}$ with $\psi=\phi_{1}$ and $\phi=\phi_{r}$ such that each $\phi_{i+1}$ is obtained from $\phi_{i}$ by knot insertion, see Fig. 10. If $\phi \prec \psi$ we define

$$
c_{\psi, \phi}:=\prod_{i=2}^{r} a_{i}, \quad \text { where } \phi_{i-1}=a_{i} \phi_{i}+b_{i} \tilde{\phi}_{i} \text { as in } 77 \text {. }
$$

We write $\phi \prec_{\mathbb{K}} \psi$ if there exists a similar sequence whose elements are all contained in $\mathbb{K}$. The minimal B-splines for $\prec_{\mathbb{K}}$ are called minimal support B-splines in $\mathbb{K}$.

Since $\mathbb{P}_{\boldsymbol{d}} \subseteq \mathbb{K}$, the space $\mathbb{K}$ contains the Bernstein polynomials $B_{\boldsymbol{i}}, \boldsymbol{i} \in[\mathbf{0}, \boldsymbol{d}] \cap \mathbb{N}^{n}$ on $\Omega$. The LR generating set $\Phi$ associated to $\mathbb{K}$ contains the minimal support Bsplines $\phi \in \mathbb{K}$ that are obtained from the $B_{\boldsymbol{i}}$ 's using knot insertion. The B-splines in $\Phi$ span a subset of $\mathbb{K}$ containing $\mathbb{P}_{\boldsymbol{d}}$, but they can be linearly dependent. If $g \in \mathbb{P}_{\boldsymbol{d}}$ then we have $g=\sum_{\phi \in \Phi} \mathfrak{l}(g, \phi) \phi$, where, with $\mathfrak{c}(g, \phi)$ as in (67), the coefficients are given by the following recursive formula [8, Theorem 4.4]

$$
\mathfrak{l}(g, \phi):=\mathfrak{c}(g, \phi)-\sum_{\psi \in N_{\phi}} c_{\psi, \phi} \mathfrak{l}(g, \psi), \quad N_{\phi}:=\{\psi \in \Phi: \phi \prec \psi\} .
$$


Unfolding the recursion we find

$$
\mathfrak{l}(g, \phi)=\mathfrak{c}(g, \phi)+\sum_{\psi \in N_{\phi}} z_{\psi, \phi} \mathfrak{c}(g, \psi)
$$

for some $z_{\psi, \phi} \in \mathbb{R}$. It follows that $\overline{\mathrm{H}_{\mathbb{P}}}$ is satisfied by the collection $\Lambda=\left\{\lambda_{\phi}, \phi \in \Phi\right\}$

$$
\lambda_{\phi}:=S_{\phi}+\sum_{\psi \in N_{\phi}} z_{\psi, \phi} G_{\psi, \operatorname{supp} \phi}
$$

where $S_{\phi}$ and $G_{\psi, \text { supp } \phi}$ are defined in $(68)$ and $(69)$, respectively.

Theorem 10 Let $\Phi$ be an LR generating system satisfying $\mathrm{H}_{\phi}^{\Theta}, \mathrm{H}_{\mathrm{E}}$ and such that for all $\phi \in \Phi, \psi \in N_{\phi}$ we have $\boldsymbol{h}_{\psi} \leq$ l $\boldsymbol{h}_{\phi}$. Then the collection $\Lambda=\left\{\lambda_{\phi}, \phi \in \Phi\right\}$ with $\lambda_{\phi}$ as in 80 defines an operator $\aleph$ that satisfies $\mathrm{H}_{\mathbb{P}}, \mathrm{H}_{\lambda} \mathrm{H}_{\phi}$ and $\mathrm{H}_{\mathrm{s}}$ with

$$
\begin{gathered}
C_{\lambda}=2^{C_{\mathrm{E}}+|\boldsymbol{d}+\mathbf{1}|}(\boldsymbol{d}+\mathbf{1})^{\mathbf{1 - 1} / p} C_{\mathrm{E}} \ell^{|\boldsymbol{d}|+\frac{n}{p}} \prod_{i=1}^{n}\left\|H_{d_{i}}^{-1}\right\|, \\
C_{\Phi}=\frac{(\boldsymbol{d}+1) ! 2^{|\boldsymbol{\sigma}|}}{(\boldsymbol{d}-\boldsymbol{\sigma}) !} C_{\Theta}^{|\boldsymbol{\sigma}|}, \quad C_{\mathrm{s}}=1, \quad C_{\star}=1 .
\end{gathered}
$$

Moreover, if $C_{\mathrm{E}}=(\boldsymbol{d}+\mathbf{1})^{\mathbf{1}}$, then $C_{\lambda}=(\boldsymbol{d}+\mathbf{1})^{\mathbf{1}} 2^{|\boldsymbol{d}|+n}$.

Proof $\mathrm{H}_{\mathbb{P}}$ follows from (80). Proposition 10 implies $\mathrm{H}_{\phi}$. Since $\mathbf{s}_{\phi}$ is a box we deduce $\mathrm{H}_{\mathrm{s}}$ with $C_{\mathrm{s}}=1$ and $\mathrm{H}_{\star}$ with $C_{\star}=1$. We need to show $\mathrm{H}_{\lambda}$. From 80 we have

$$
\left\|\lambda_{\phi}\right\|_{* p} \leq\left(\left\|S_{\phi}\right\|_{* p}+\sum_{\psi \in N_{\phi}}\left|z_{\phi, \psi}\right|\left\|G_{\psi, \operatorname{supp} \phi}\right\|_{* p}\right) .
$$

The $z_{\psi, \phi}$ are sums of products of $c_{\eta, \beta}$. Consequently they are sums of terms in $[-1,1]$. Therefore $\left|z_{\psi, \phi}\right|$ is bounded by the number of terms and we have

$$
\left|z_{\psi, \phi}\right| \leq \#\left\{\text { oriented paths in } N_{\phi} \text { from } \psi \text { to } \phi\right\} \leq 2^{\# N_{\phi}-1} \leq 2^{C_{\mathrm{E}}-2}
$$

Finally, $\phi \prec \psi$ implies $\|\phi\|_{p} \leq\|\psi\|_{p}$, and using (81), (82), Proposition 12 and $\boldsymbol{h}_{\psi} \leq \ell \boldsymbol{h}_{\phi}$ we obtain $\mathrm{H}_{\lambda}$ with the claimed $C_{\lambda}$.

If $C_{\mathrm{E}}=(\boldsymbol{d}+\mathbf{1})^{\mathbf{1}}$ then by [10, Theorem 4], $N_{\phi}=\emptyset$ and $\lambda_{\phi}=S_{\phi}$.

\section{Closing remarks}

The polynomial approximation assumption $\mathrm{H}_{\Pi}$ can be extended to allow for more general bounding terms. A possibility are fractional order Sobolev seminorms, or derivative dependent summability $q_{k}$ as in 17 . We do not foresee major difficulties in such extensions, but surely they would be notationally heavy.

A second remark is that the results are based on local bounds. In particular the cardinality of $\Phi$ and the boundedness of $\Omega$ are not used in the proofs. Note however that the embedding of $L^{q}(\Omega) \subseteq L^{p}(\Omega)$ for the case $p \leq q$ requires $\mu(\Omega)<\infty$. 
Assumptions for space independent estimates

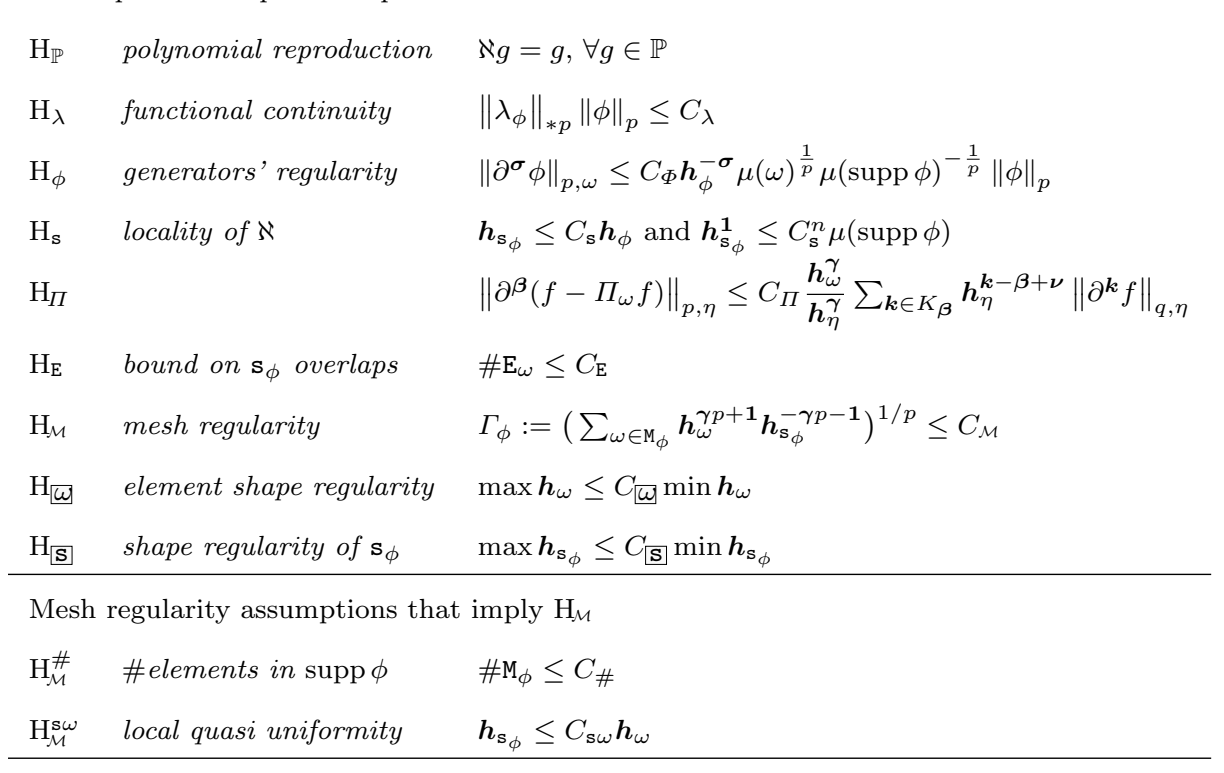

Assumptions that imply $\mathrm{H}_{\Pi}$ using averaged Taylor expansion

$\mathrm{H}_{\star} \quad \mathbf{s}_{\phi}$ star shaped and $\boldsymbol{h}_{\mathbf{s}_{\phi}} \leq C_{\star} \boldsymbol{h}_{\zeta_{\phi}}$

B-spline specific assumption

$\mathrm{H}_{\phi}^{\Theta} \quad$ knot vector regularity $\quad \Delta_{\phi, i, d_{i}+1} \Delta_{\phi, i, d_{i}-\sigma_{i}+1}^{-1} \leq C_{\Theta}$

Table 1 Table of the assumptions and corresponding constants.

\section{Acknowledgement}

The first author has received funding from the European Research Council under the European Union's Seventh Framework Programme (FP7/2007-2013) / ERC grant agreement 339643 .

\section{References}

1. Adams, R.A., Fournier, J.J.F.: Sobolev spaces, Pure and Applied Mathematics (Amsterdam), vol. 140, second edn. Elsevier/Academic Press, Amsterdam (2003)

2. Beckenbach, E.F., Bellman, R.: Inequalities, fourth edn. Ergebnisse der Mathematik und ihrer Grenzgebiete, N. F., Bd. 30. Springer-Verlag, Berlin (1983)

3. Beirão da Veiga, L., Buffa, A., Cho, D., Sangalli, G.: Analysis-suitable T-splines are dualcompatible. Comput. Methods Appl. Mech. Engrg. 249/252, 42-51 (2012). DOI 10.1016/ j.cma.2012.02.025

4. Beirão da Veiga, L., Buffa, A., Sangalli, G., Vázquez, R.: Analysis-suitable T-splines of arbitrary degree: definition, linear independence and approximation properties. Math. Models Methods Appl. Sci. 23(11), 1979-2003 (2013). DOI 10.1142/S0218202513500231

5. Beirão da Veiga, L., Buffa, A., Sangalli, G., Vázquez, R.: Mathematical analysis of variational isogeometric methods. Acta Numerica 23, 157-287 (2014). DOI 10.1017/ S096249291400004X 
6. Beirão da Veiga, L., Cho, D., Sangalli, G.: Anisotropic NURBS approximation in isogeometric analysis. Comput. Methods Appl. Mech. Engrg. 209/212, 1-11 (2012). DOI 10.1016/j.cma.2011.10.016

7. de Boor, C.: A practical guide to splines, Applied Mathematical Sciences, vol. 27, revised edn. Springer-Verlag, New York (2001)

8. Bressan, A.: Some properties of LR-splines. Comput. Aided Geom. Design 30(8), 778-794 (2013)

9. Bressan, A., Buffa, A., Sangalli, G.: Characterization of analysis-suitable T-splines. Comput. Aided Geom. Design 39, 17-49 (2015). DOI 10.1016/j.cagd.2015.06.007

10. Bressan, A., Jüttler, B.: A hierarchical construction of LR meshes in 2D. Comput. Aided Geom. Design 37, 9-24 (2015). DOI 10.1016/j.cagd.2015.06.002

11. Bressan, A., Mokriš, D.: A versatile strategy for the implementation of adaptive splines. In: Mathematical methods for curves and surfaces, Lecture Notes in Comput. Sci., vol. 10521, pp. 42-73. Springer, Cham (2017)

12. Buffa, A., Garau, E.M., Giannelli, C., Sangalli, G.: On quasi-interpolation operators in spline spaces. In: Building bridges: connections and challenges in modern approaches to numerical partial differential equations, Lect. Notes Comput. Sci. Eng., vol. 114, pp. 73-91. Springer, [Cham] (2016)

13. Buffa, A., Giannelli, C.: Adaptive isogeometric methods with hierarchical splines: error estimator and convergence. Math. Models Methods Appl. Sci. 26(1), 1-25 (2016). DOI 10.1142/S0218202516500019

14. Buffa, A., Giannelli, C., Morgenstern, P., Peterseim, D.: Complexity of hierarchical refinement for a class of admissible mesh configurations. Comput. Aided Geom. Design 47, 83-92 (2016). DOI 10.1016/j.cagd.2016.04.003

15. Buffa, A., Sangalli, G., Schwab, C.: Exponential convergence of the $h p$ version of isogeometric analysis of 1D. In: Spectral and high order methods for partial differential equationsICOSAHOM 2012, Lect. Notes Comput. Sci. Eng., vol. 95, pp. 191-203. Springer, Cham (2014). DOI 10.1007/978-3-319-01601-6_15

16. Dokken, T., Lyche, T., Pettersen, K.F.: Polynomial splines over locally refined boxpartitions. Comput. Aided Geom. Design 30(3), 331-356 (2013). DOI 10.1016/j.cagd. 2012.12.005

17. Dupont, T., Scott, R.: Polynomial approximation of functions in Sobolev spaces. Math. Comp. 34(150), 441-463 (1980). DOI 10.2307/2006095

18. Durán, R.G.: On polynomial approximation in Sobolev spaces. SIAM J. Numer. Anal. 20(5), 985-988 (1983). DOI 10.1137/0720068

19. Forsey, D.R., Bartels, R.H.: Hierarchical B-spline refinement. SIGGRAPH Comput. Graph. 22(4), 205-212 (1988). DOI 10.1145/378456.378512

20. Giannelli, C., Jüttler, B., Speleers, H.: THB-splines: the truncated basis for hierarchical splines. CAGD 29(7), 485-498 (2012). DOI 10.1016/j.cagd.2012.03.025

21. Giannelli, C., Jüttler, B., Speleers, H.: Strongly stable bases for adaptively refined multilevel spline spaces. Adv. Comput. Math. 40(2), 459-490 (2014). DOI 10.1007/ s10444-013-9315-2

22. Hughes, T.J.R., Cottrell, J.A., Bazilevs, Y.: Isogeometric analysis: CAD, finite elements, NURBS, exact geometry and mesh refinement. Comput. Methods Appl. Mech. Engrg. 194(39-41), 4135-4195 (2005)

23. Kraft, R.: Adaptive and linearly independent multilevel B-splines. In: A. Le Méhauté, C. Rabut, L.L. Schumaker (eds.) Surface Fitting and Multiresolution Methods, pp. 209218. Vanderbilt University Press, Nashville (1997)

24. Li, X., Zheng, J., Sederberg, T.W., Hughes, T.J.R., Scott, M.A.: On linear independence of T-spline blending functions. Comput. Aided Geom. Design 29(1), 63-76 (2012). DOI 10.1016/j.cagd.2011.08.005

25. Lyche, T., Manni, C., Speleers, H.: Foundations of spline theory: B-splines, spline approximation, and hierarchical refinement. In: T. Lyche, C. Manni, H. Speleers (eds.) Splines and PDEs: From Approximation Theory to Numerical Linear Algebra, Cetraro, Italy 2017, vol. 2219, pp. 1-76. Springer Nature Switzerland AG (2018). DOI 10.1007/978-3-319-94911-6

26. Lyche, T., Mørken, K.M.: Spline Methods Draft (2011)

27. Lyche, T., Schumaker, L.L.: Local spline approximation methods. J. Approx. Theory 15, 294-325 (1975). DOI 10.1016/0021-9045(75)90091-X

28. Mokriš, D., Jüttler, B.: TDHB-splines: the truncated decoupled basis of hierarchical tensorproduct splines. Comput. Aided Geom. Design 31(7-8), 531-544 (2014). DOI 10.1016/j. cagd.2014.05.004 
29. Morgenstern, P.: Globally structured three-dimensional analysis-suitable T-splines: definition, linear independence and $m$-graded local refinement. SIAM J. Numer. Anal. 54(4), 2163-2186 (2016). DOI 10.1137/15M102229X

30. Morgenstern, P., Peterseim, D.: Analysis-suitable adaptive T-mesh refinement with linear complexity. Comput. Aided Geom. Design 34, 50-66 (2015). DOI 10.1016/j.cagd.2015. 02.003

31. Range, R.M.: Holomorphic functions and integral representations in several complex variables, Graduate Texts in Mathematics, vol. 108. Springer-Verlag, New York (1986). DOI 10.1007/978-1-4757-1918-5

32. Reif, U.: Polynomial approximation on domains bounded by diffeomorphic images of graphs. J. Approx. Theory 164(7), 954-970 (2012). DOI 10.1016/j.jat.2012.03.010

33. Reif, U., Sissouno, N.: Approximation with diversified B-splines. Comput. Aided Geom. Design 31(7-8), 510-520 (2014)

34. Scherer, K., Shadrin, A.: New upper bound for the b-spline basis condition number: Ii. a proof of de boor's 2k-conjecture. Journal of approximation theory 99(2), 217-229 (1999)

35. Schötzau, D., Schwab, C.: Exponential convergence for $h p$-version and spectral finite element methods for elliptic problems in polyhedra. Math. Models Methods Appl. Sci. 25(9), 1617-1661 (2015). DOI 10.1142/S0218202515500438

36. Schumaker, L.L.: Spline functions: basic theory, third edn. Cambridge Mathematical Library. Cambridge University Press, Cambridge (2007). DOI 10.1017/CBO9780511618994

37. Sederberg, T.W., Cardon, D.L., Finnigan, G.T., North, N.S., Zheng, J., Lyche, T.: T-spline simplification and local refinement. In: ACM transactions on graphics (TOG), vol. 23, pp. 276-283. ACM (2004)

38. Sederberg, T.W., Zheng, J., Bakenov, A., Nasri, A.: T-splines and T-NURCCs. In: ACM transactions on graphics (TOG), vol. 22, pp. 477-484. ACM (2003)

39. Speleers, H.: Hierarchical spline spaces: quasi-interpolants and local approximation estimates. Adv. Comput. Math. 43(2), 235-255 (2017). DOI 10.1007/s10444-016-9483-y

40. Speleers, H., Manni, C.: Effortless quasi-interpolation in hierarchical spaces. Numer. Math. 132(1), 155-184 (2016). DOI 10.1007/s00211-015-0711-z

41. Verfürth, R.: A note on polynomial approximation in Sobolev spaces. M2AN Math. Model. Numer. Anal. 33(4), 715-719 (1999). DOI 10.1051/m2an:1999159 